\title{
INFLUENCE OF SOME SEEDING AND NITROGEN RATES ON GRAIN YIELD AND INSECT NATURAL INFESTATION OF SOME WHEAT CULTIVARS
}

\author{
KHALED, M.A. ${ }^{1}$ and A.M. EL-RAWY ${ }^{2}$ \\ 1- Wheat Department, Field Crops Research Institute, ARC, Giza, Egypt. \\ 2- Plant Protection Research Institute, ARC, Dokki, Giza, Egypt.
}

(Manuscript received 27 March 2012)

\begin{abstract}
A Field experiment was performed on Bahteem Agric. Res. Station Farm Qalubia Governorate, during the two growing seasons $2009 / 10$ and $2010 / 11$ to study the effect of three seeding rates i.e. 300,350 and 400 grains $/ \mathrm{m}^{2}$ and two nitrogen fertilizer levels i.e. 75 and $90 \mathrm{~kg} \mathrm{~N} /$ fed on yield and yield components as well as grain crude protein and total carbohydrate percentages. In addition the intensity of natural infestation with cereal aphids and leafminer on six wheat cultivars i.e. Sakha 93, Sakha 94, Gemmeiza 10 and Sids 12 (bread wheat); Beni-Sweif 4 and Beni-Sweif 5 (durum wheat) was also investigated.

Results indicated that Beni-Sweif 4 cultivar exhibited longer period to heading (89.5 days) and to maturity (140.5 days). On the other hand, Beni-Sweif 5 exhibited taller plants $(101.2 \mathrm{~cm})$ followed by Beni-Sweif $4(99.9 \mathrm{~cm})$ and Sids $12(97.5 \mathrm{~cm})$. While Beni-Sweif 5 and Sids 12 cultivars gave more spikes (518.5 and 516.1 spikes/ $\mathrm{m}^{2}$, respectively), but highest number of grains /spike was shown by Beni-Sweif 5 and Beni-Sweif 4 (67.4 and 66.0 grains/spike, respectively).The highest values of 1000 -grain weight and grain yield were in Beni-Sweif 4 and Sids 12 (48.6 and $48.5 \mathrm{~g} \& 16.2$ and $16.3 \mathrm{ardab} / \mathrm{fed}$., respectively). While Beni-Sweif 4 gave the highest value of straw yield (3.6 ton/fed.). On the other hand, Beni-Sweif 5 and Beni-Sweif 4 cultivars gave the highest values of grain crude protein and total carbohydrate percentages (12.2 and $11.9 \%$ \& 76.7 and $75.8 \%$, respectively). As seeding rate increased caused a significant increase in period to heading (90 days) and to maturity (138 days), number of spikes $/ \mathrm{m}^{2}$ (522.1 spikes $/ \mathrm{m}^{2}$ ) and straw yield (3.5 ton /fed.) and caused a significant decrease in plant height $(89.6 \mathrm{~cm})$, number of grains/spike (56.6 grains/spike), 1000-grain weight $(42.9 \mathrm{~g})$, grain yield (15.5 ardab/fed.) and also decrease in grain crude protein and total carbohydrate percentages (11 and $71.3 \%$,respectively). Seeding rate of $300 \mathrm{grains} / \mathrm{m}^{2}$ was caused a significant increase in plant height $(101.4 \mathrm{~cm})$, number of grains /spike (64.8 grains/spike), 1000-grain weight (49.2 g), grain yield (16.2 ardab /fed.) and also caused increase in grain crude protein and total carbohydrate percentages (11.8 and $73.2 \%$,respectively).
\end{abstract}


Increasing nitrogen rate from 75 to $90 \mathrm{Kg} /$ fed caused a significant increase in plant height $(97.3 \mathrm{~cm})$, number of spikes $/ \mathrm{m}^{2}(514.7$ spikes $\left./ \mathrm{m}^{2}\right)$, number of grains /spike (61.5 grains/spike), 1000-grain weight (46.8 g), grain yield (16.2 ardab /fed.), straw yield (3.4 ton (fed.) and also caused increase in grain crude protein and total carbohydrate percentages (11.5 and $72.6 \%$,respectively).

For insect infestation, results revealed that Beni-Sweif 4 and Sids 12 cultivars were significantly effective in decreasing number of cereal aphids (62.1 and 83.3 aphids /10 plants, respectively). On the other hand, Beni-Sweif 4 and Beni-Sweif 5 showed the least number of leafminer (2.4 and 3.4 mines \& 1.5 and 2.2 larvae /100 leaves, respectively). Increasing seeding rate from $300 \mathrm{grains} / \mathrm{m}^{2}$ to 400 grains $/ \mathrm{m}^{2}$ caused a significant increase in number of cereal aphids (159.5 aphids /10 plants) and leafminer (9.2 mines and 6.7 larvae /100 leaves). The least number of cereal aphids (82.1 aphids /10 plants) and leafminer (3.7 mines and 2.5 larvae /100 leaves) were obtained at a seeding rate of 300 grains $/ \mathrm{m}^{2}$. Increasing nitrogen rate from 75 to $90 \mathrm{Kg} /$ fed caused a significant increase in number of cereal aphids (133.3 aphids /10 plants) and leafminer (7.9 mines and 5.6 larvae /100 leaves).

From these results, it could be concluded that sowing Sids 12 cultivar (bread wheat) or Beni-Sweif 4 cultivar (durum wheat) under seeding rate of 300 grains $/ \mathrm{m}^{2}$ and applying $90 \mathrm{KgN} /$ fed gave the highest grain yield/fed and least infestation by cereal aphids and leafminer.

\section{INTRODUCTION}

Wheat (Triticum aestivum L.) is the most important cereal crop in the world. Because of the difficulties facing the horizontal expansion for production increase in Egypt, the vertical yield increase is the main possible mean which could be achieved by developing high yielding varieties and via good cultural practices i.e. optimum seeding rate, fertilization and pest management (El-Ghareib et. al.. 1998, El-Karamity, 1998, Munir et. al.., 2001, Abdel-Aziz et. al.., 2002, SIman, 2002, SIman et. al.., 2002, Mousa, 2006, El-Rawy et. al.., 2007, Swelam, 2008 and Abdel-Nour and Fateh, 2011). During the last few years, cereal aphids have become serious insect pests attacking wheat plants in Egypt. These pests cause a serious damage to the plants either directly by sucking plant cell juice or indirectly as a vector of diseases (Abdel-Aziz et. al.., 2002 and SIman, 2002). The leafminer Agromyza nigrella (Rondani) attacks wheat in Egypt in a range of 2 - 90\% infestation (El-Serwy, 1996 and SIman et. al.., 2002). However, aphids and leafminer are considered two of the important pests 
attacking wheat plants and causing sever reduction in the yield (SIman, 2002 and SIman et. al., 2002).

This work aimed to study the effect of seeding and nitrogen fertilizer rates on yield, yield components as well as chemical content of wheat grains under infestation with cereal aphids and leafminer of six wheat cultivars.

\section{MATERIALS AND METHODS}

A Field experiment was carried out at Bahteem Agricultural Research Station, Qalubia Governorate during two successive wheat seasons of 2009/10 and 2010/11. The experiments were sown on December $6^{\text {th }}, 2009$ and November $27^{\text {th }}, 2010$ using a split-split plot design with four replications. Six wheat cultivars were distributed at random in the main plots, four of these cultivars were bread wheat (Sakha 93, Sakha 94, Gemmeiza 10 and Sids 12) and the other two were durum wheat (Beni-Sweif 4 and Beni-Sweif 5). Three seeding rates $\left(300,350\right.$ and $\left.400 \mathrm{grains} / \mathrm{m}^{2}\right)$ were assigned to the sub-plots. Two nitrogen fertilizer rates ( 75 and $90 \mathrm{KgN} / \mathrm{fed}$.) were allocated to the sub-sub plots. Nitrogen was added in the form of ammonium nitrate $(33.5 \% \mathrm{~N})$ at three doses i.e. $20 \%$ at sowing, $40 \%$ at tillering and $40 \%$ at elongation stage. The area of sub-sub plot was $11.4 \mathrm{~m}^{2}(3.8 \times 3 \mathrm{~m})$, each plot contained 19 rows with $20 \mathrm{~cm}$ apart.

Agronomic data recorded in the two growing seasons were: days to heading and to maturity, plant height, number of spikes $/ \mathrm{m}^{2}$, number of grains/spike, 1000grain weight and grain and straw yields. Chemical constituents of wheat grains for protein and total carbohydrate content were determined according to the A.O.A.C. (2000).

To determine aphid population and leafminer infestation, weekly samples each of ten plants, starting from $1^{\text {st }}$ Feb. to $1^{\text {st }}$ April were picked up at random from each sub-sub plot to record number of cereal aphid (Rhopalosiphum padi, Schizaphis graminum, Sitobion avenae and Rhopalosiphum maidis) and, at the same time, number of Agromyza nigrella mines and alive larvae / 25 leaves.

Data obtained in each season were statistically analysed according to procedures outlined by Steel et. al. (1997) and the treatment means were compared by Least Significant Difference (L.S.D.) at $5 \%$ level. 


\section{RESULTS AND DISCUSSION}

Results presented in Tables (1 to 8 ) show the effects of seeding and nitrogen fertilizer rates on yield, yield components and chemical of wheat grain content and infestation with cereal aphids and leafminer of six wheat cultivars.

\section{1- Effects on the yield}

\subsection{Cultivar performance}

Data presented in Tables ( 1 to 5 ) showed significant differences among the tested cultivars for all the studied wheat traits. Beni-Sweif 4 cultivar gave the highest values for days to heading and to maturity, 1000-grain weight, grain and straw yields/fed (89.5 and 140.5 days, $48.6 \mathrm{~g}, 16.2 \mathrm{ardab} /$ fed and 3.6 ton/fed., respectively ), while Sakha 93 cultivar gave the lowest values for number of spikes $/ \mathrm{m}^{2}$, number of grains /spike, 1000-grain weight and grain and straw yields (493.4 spikes/m², 54 grains /spike, 43.7g, 15.5 ardab/fed and 3.3 ton/fed ., respectively). Sids 12 (bread wheat) and Beni-Sweif 4 (durum wheat) cultivars gave the highest values for 1000grain weight and grain yield/fed (48.5 and $48.6 \mathrm{~g} \& 16.3$ and 16.2 ardab/fed., respectively). The differences in 1000-grain weight and grain yield between Sids 12 and Beni-Sweif 4 cultivars were insignificant. Beni-Sweif 5 (durum wheat) gave the highest values of plant height, number of spikes $/ \mathrm{m}^{2}$, number of grains/spike, protein and total carbohydrate percentages $\left(101.2 \mathrm{~cm}, 518.5\right.$ spikes $/ \mathrm{m}^{2}, 67.4$ grains/spike, $12.2 \%$ and $76.7 \%$, respectively). These results are in agreement with those obtained by El-Ghareib et. al. (1998), El-Karamity (1998), Sharaan and Abdel-Samie (1999), El-Naggar (2003), Mousa (2006) and Abdel-Nour and Fateh (2011).

\subsection{Seeding rates}

Data compiled in Tables (1 to 5 ) show significant increase in days to heading and to maturity, number of spikes $/ \mathrm{m}^{2}$ and straw yield/fed with increasing seeding rates. Whereas, seeding rate at 300 grains $/ \mathrm{m}^{2}$ gave the highest values for plant height, number of grains/spike, 1000-grain weight, grain yield, protein and total carbohydrate percentages (101.4 cm, 64.8 grains/spike, 49.2g, 16.2 ardab/fed., $11.8 \%$ and $73.2 \%$, respectively) as compared with the two higher seeding rates. While increasing seeding rates from 300 grains $/ \mathrm{m}^{2}$ to 400 grains $/ \mathrm{m}^{2}$ caused increase 
in days to heading and to maturity, number of spikes $/ \mathrm{m}^{2}$ and straw yield ( 90 and 138 days , 522.1 spikes/ $\mathrm{m}^{2}$ and 3.5 ton/fed .,respectively) and caused a significant decease in plant height, number of grains/spike, 1000-grain weight, grain yield and also decrease in grain crude protein and total carbohydrate percentages $(89.6 \mathrm{~cm}$, 56.6 grains/spike, $42.9 \mathrm{~g}, 15.5$ ardab/fed., $11 \%$ and $71.3 \%$, respectively). Beni-Sweif 4 cultivar (durum wheat) and Sids 12 cultivar (bread wheat) gave the highest grain yield (16.8 and 16.6 ardab/fed., respectively) when sown with a rate of 300 grains/ $\mathrm{m}^{2}$, while Beni-Sweif 4 and Beni-Sweif 5 cultivars (durum wheat) and Sakha 94 cultivar (bread wheat) gave the highest straw yield (3.6, 3.5 and 3.5 ton/fed., respectively) when the seeding rate was increased to 400 grains/ $\mathrm{m}^{2}$. Comparable data have been reported by Hefni et. al. (1979), El-Ghareib et. al. (1998), El-Karamity (1998), Sharaan and Abdel-Samie (1999), Munir et. al. (2001) and Mousa (2006).

\subsection{Nitrogen rates}

As shown in Tables (1 to 5), significant increase in all studied characters were studied with increasing nitrogen rates. The highest values for days to heading and to maturity, plant height, number of spikes $/ \mathrm{m}^{2}$, number of grains/spike, 1000-grain weight, grain yield, straw yield, protein and total carbohydrate percentages were 89 and 137days, $97.3 \mathrm{~cm}, 514.7$ spikes $/ \mathrm{m}^{2}, 61.5$ grains/spike, $46.8 \mathrm{~g}, 16.2$ ardab/fed., 3.4 ton/fed., $11.5 \%$ and $72.6 \%$, respectively. Beni-Sweif 5 and Beni-Sweif 4 cultivars (durum wheat) gave highest values for plant height $(101.2$ and $99.9 \mathrm{~cm}$, respectively), number of spikes $/ \mathrm{m}^{2}$ (537.8 and 522.6 spikes $/ \mathrm{m}^{2}$, respectively), number of grains/spike (68.5 and 66.4 grains/spike, respectively), 1000-grain weight (49 g), protein (12.2 and 12\%, respectively) and total carbohydrate percentages (76.8 and $76.1 \%$, respectively) at the highest nitrogen rate $(90 \mathrm{KgN} / \mathrm{fed}$.). While Sids 12 (bread wheat) and Beni-Sweif 4 (durum wheat) gave the highest value for grain yield (16.5 ardab/fed), but Beni-Sweif 4 and Beni-Sweif 5 (durum wheat) and Sakha 94 (bread wheat) gave the highest values for straw yield (3.4, 3.3 and 3.3 ton/fed., respectively) at the highest nitrogen rate $(90 \mathrm{KgN} / \mathrm{fed}$.). Similar results were reported by El-Ghareib et. al.. (1998), El-Karamity (1998), Sharaan and Abdel-Samie (1999), Munir (2001), El-Naggar (2003), El-Rawy et. al. (2007), Swelam (2008) and Abdel-Nour and Fateh (2011). 
Table 1. Effect of seeding and nitrogen fertilizer rates on days to heading and to maturity of some wheat cultivars during the two successive seasons (2009/10 and 2010/11).

\begin{tabular}{|c|c|c|c|c|c|c|c|c|c|c|c|c|c|c|c|c|c|c|c|c|c|c|c|c|c|c|c|c|}
\hline \multirow{5}{*}{ Cultivar } & & \multicolumn{27}{|c|}{ Days to heading } \\
\hline & & \multicolumn{9}{|c|}{$2009 / 10$} & \multicolumn{9}{|c|}{$2010 / 11$} & \multicolumn{9}{|c|}{ Mean } \\
\hline & & \multicolumn{9}{|c|}{ Seeding rate } & \multicolumn{9}{|c|}{ Seeding rate } & \multicolumn{9}{|c|}{ Seeding rate } \\
\hline & & \multicolumn{2}{|c|}{ 300grains $/ \mathrm{m}^{2}$} & \multicolumn{2}{|c|}{ 350grains $/ \mathrm{m}^{2}$} & \multicolumn{2}{|c|}{ 400grains $/ \mathrm{m}^{2}$} & \multicolumn{2}{|c|}{ Mean } & \multirow{2}{*}{$\begin{array}{c}\text { General } \\
\text { mean }\end{array}$} & \multicolumn{2}{|c|}{ 300grains $/ \mathrm{m}^{2}$} & \multicolumn{2}{|c|}{ 350grains $/ \mathrm{m}^{2}$} & \multicolumn{2}{|c|}{ 400grains $/ \mathrm{m}^{2}$} & \multicolumn{2}{|c|}{ Mean } & \multirow{2}{*}{$\begin{array}{c}\text { General } \\
\text { mean }\end{array}$} & \multicolumn{2}{|c|}{ 300grains $/ \mathrm{m}^{2}$} & \multicolumn{2}{|c|}{ 350grains/m² } & \multicolumn{2}{|c|}{ 400grains $/ \mathrm{m}^{2}$} & \multicolumn{2}{|c|}{ Mean } & \multirow{2}{*}{$\begin{array}{c}\text { General } \\
\text { mean }\end{array}$} \\
\hline & & $75 \mathrm{KgN}$ & $90 \mathrm{KgN}$ & $75 \mathrm{KgN}$ & $90 \mathrm{KgN}$ & $75 \mathrm{KgN}$ & $90 \mathrm{KgN}$ & $75 \mathrm{KgN}$ & $90 \mathrm{KgN}$ & & $75 \mathrm{KgN}$ & $90 \mathrm{KgN}$ & $75 \mathrm{KgN}$ & $90 \mathrm{KgN}$ & $75 \mathrm{KgN}$ & $90 \mathrm{KgN}$ & $75 \mathrm{KgN}$ & $90 \mathrm{KgN}$ & & $75 \mathrm{KgN}$ & $90 \mathrm{KgN}$ & $75 \mathrm{KgN}$ & $90 \mathrm{KgN}$ & $75 \mathrm{KgN}$ & $90 \mathrm{KgN}$ & $75 \mathrm{KgN}$ & $90 \mathrm{KgN}$ & \\
\hline Sakha 93 & & 83 & 86 & 85 & 86 & 90 & 91 & 86 & 87 & $86.5 \mathrm{e}$ & 84 & 85 & 85 & 86 & 89 & 91 & 86 & 87 & $86.5 \mathrm{c}$ & 84 & 85 & 85 & 86 & 90 & 91 & 86 & 87 & $86.5 \mathrm{e}$ \\
\hline Sakha 94 & ర్ల & 85 & 87 & 87 & 88 & 89 & 92 & 87 & 89 & $88.0 \mathrm{~d}$ & 84 & 85 & 85 & 86 & 87 & 90 & 85 & 87 & $86.0 \mathrm{~cd}$ & 84 & 86 & 86 & 87 & 88 & 91 & 86 & 88 & $87.0 \mathrm{~d}$ \\
\hline Gemmeiza10 & $\frac{\mathscr{\omega}}{\bar{\omega}} \frac{\frac{c}{3}}{3}$ & 86 & 88 & 87 & 90 & 91 & 92 & 88 & 90 & $89.0 \mathrm{~b}$ & 87 & 88 & 88 & 89 & 90 & 91 & 88 & 89 & $88.5 \mathrm{a}$ & 87 & 88 & 88 & 90 & 91 & 92 & 89 & 90 & $89.5 \mathrm{~b}$ \\
\hline Sids 12 & & 84 & 87 & 85 & 87 & 86 & 89 & 85 & 88 & $86.5 \mathrm{e}$ & 84 & 85 & 86 & 86 & 86 & 88 & 85 & 86 & $85.5 \mathrm{~d}$ & 84 & 86 & 86 & 86 & 86 & 89 & 85 & 87 & $86.0 \mathrm{f}$ \\
\hline $\begin{array}{c}\text { Beni- } \\
\text { Sweif } 4\end{array}$ & 巨蛋 & 87 & 88 & 91 & 91 & 91 & 92 & 89 & 90 & $89.5 a$ & 87 & 88 & 90 & 89 & 91 & 91 & 89 & 89 & $89.0 \mathrm{a}$ & 87 & 88 & 90 & 90 & 91 & 92 & 89 & 90 & $89.5 a$ \\
\hline $\begin{array}{c}\text { Beni- } \\
\text { Sweif } 5\end{array}$ & 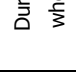 & 85 & 87 & 89 & 90 & 90 & 92 & 88 & 90 & $89.0 \mathrm{c}$ & 86 & 86 & 89 & 89 & 90 & 92 & 88 & 89 & $88.5 b$ & 86 & 87 & 89 & 90 & 90 & 92 & 88 & 90 & $89.0 \mathrm{c}$ \\
\hline Mean & & 85 & 87 & 87 & 89 & 90 & 91 & $87 \mathrm{~b}$ & $89 a$ & - & 85 & 86 & 87 & 88 & 89 & 91 & $87 \mathrm{~b}$ & $88 a$ & - & 85 & 87 & 97 & 88 & 89 & 91 & $87 \mathrm{~b}$ & $89 a$ & - \\
\hline General mear & & 86.0 & & 88. & $\mathrm{ob}$ & 90. & $5 \mathrm{a}$ & & - & & 85. & & 87. & & 90 & $.0 \mathrm{a}$ & & - & & 86. & $.0 \mathrm{c}$ & 87. & $.5 b$ & 90. & $.0 \mathrm{a}$ & & - & \\
\hline Interaction & & & & & & N.S. & & & & & & & & & N.S. & & & & & & & & & N.S. & & & & \\
\hline & & & & & & & & & & & & Days & s to mat & turity & & & & & & & & & & & & & & \\
\hline Sakha 93 & & 130 & 131 & 131 & 132 & 135 & 136 & 132 & 133 & $132.5 \mathrm{e}$ & 129 & 130 & 130 & 132 & 134 & 135 & 131 & 132 & $131.5 \mathrm{e}$ & 129 & 131 & 131 & 132 & 135 & 136 & 132 & 133 & $132.5 \mathrm{e}$ \\
\hline Sakha 94 & 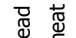 & 130 & 132 & 131 & 132 & 133 & 136 & 131 & 133 & $132.0 \mathrm{e}$ & 128 & 130 & 129 & 131 & 132 & 136 & 130 & 132 & 131.of & 129 & 131 & 130 & 132 & 133 & 136 & 131 & 133 & $132.0 f$ \\
\hline Gemmeiza10 & $\frac{5}{10} \frac{1}{3}$ & 135 & 136 & 136 & 138 & 138 & 139 & 136 & 138 & $137.0 \mathrm{c}$ & 136 & 137 & 136 & 138 & 138 & 140 & 137 & 138 & $137.5 \mathrm{c}$ & 135 & 137 & 136 & 138 & 138 & 140 & 136 & 138 & $137.0 \mathrm{c}$ \\
\hline Sids 12 & & 131 & 131 & 132 & 133 & 132 & 143 & 132 & 136 & $134.0 \mathrm{~d}$ & 130 & 133 & 132 & 133 & 134 & 136 & 132 & 134 & $133.0 \mathrm{~d}$ & 131 & 132 & 132 & 133 & 133 & 140 & 132 & 135 & $133.5 \mathrm{~d}$ \\
\hline $\begin{array}{c}\text { Beni- } \\
\text { Sweif } 4\end{array}$ & E气 芯 & 139 & 140 & 140 & 141 & 140 & 142 & 140 & 141 & $140.5 \mathrm{a}$ & 138 & 139 & 140 & 141 & 141 & 142 & 140 & 141 & $140.5 \mathrm{a}$ & 139 & 140 & 140 & 141 & 141 & 142 & 140 & 141 & $140.5 \mathrm{a}$ \\
\hline $\begin{array}{c}\text { Beni- } \\
\text { Sweif } 5\end{array}$ & & 136 & 136 & 140 & 141 & 140 & 142 & 139 & 140 & $139.5 \mathrm{~b}$ & 136 & 136 & 140 & 141 & 140 & 142 & 139 & 140 & $139.5 \mathrm{~b}$ & 136 & 136 & 140 & 141 & 140 & 142 & 139 & 140 & $139.5 b$ \\
\hline Mean & & 133 & 134 & 135 & 136 & 136 & 140 & $135 \mathrm{~b}$ & 137a & - & 133 & 134 & 135 & 136 & 137 & 139 & $135 \mathrm{~b}$ & 136a & - & 133 & 135 & 135 & 136 & 137 & 139 & $135 \mathrm{~b}$ & $137 a$ & - \\
\hline General mear & & 133. & & 135 & $.5 \mathrm{~b}$ & 138 & $.0 \mathrm{a}$ & & - & & 133 & $3.5 \mathrm{c}$ & 135. & $5.5 \mathrm{~b}$ & 138 & $3.0 \mathrm{a}$ & & - & & 134 & $4.0 \mathrm{c}$ & 135 & $5.5 \mathrm{~b}$ & 138 & $8.0 \mathrm{a}$ & & - & \\
\hline Interaction & & & & & & N.S. & & & & & & & & & N.S. & & & & & & & & & N.S. & & & & \\
\hline
\end{tabular}

Means followed by the same letter (s) in each column within each treatment are not significant. 
Table 2. Effect of seeding and nitrogen fertilizer rates on plant height and number of spikes/m2of some wheat cultivars during the two successive seasons (2009/10 and 2010/11).

\begin{tabular}{|c|c|c|c|c|c|c|c|c|c|c|c|c|c|c|c|c|c|c|c|c|c|c|c|c|c|c|c|c|}
\hline \multirow{5}{*}{ Cultivar } & & \multicolumn{27}{|c|}{ Plant height $(\mathrm{cm})$} \\
\hline & & \multicolumn{9}{|c|}{$2009 / 10$} & \multirow{2}{*}{\multicolumn{9}{|c|}{$\begin{array}{c}2010 / 11 \\
\text { Seeding rate }\end{array}$}} & \multicolumn{9}{|c|}{ Mean } \\
\hline & & \multicolumn{9}{|c|}{ Seeding rate } & & \multicolumn{7}{|c|}{ Seeding rate } & & \multicolumn{9}{|c|}{ Seeding rate } \\
\hline & & \multicolumn{2}{|c|}{ 300grains $/ \mathrm{m}^{2}$} & \multicolumn{2}{|c|}{ 350grains $/ \mathrm{m}^{2}$} & \multicolumn{2}{|c|}{ 400grains $/ \mathrm{m}^{2}$} & \multicolumn{2}{|c|}{ Mean } & \multirow{2}{*}{$\begin{array}{c}\text { General } \\
\text { mean }\end{array}$} & \multicolumn{2}{|c|}{ 300grains $/ \mathrm{m}^{2}$} & \multicolumn{2}{|c|}{350 grains $/ \mathrm{m}^{2}$} & \multicolumn{2}{|c|}{ 400grains $/ \mathrm{m}^{2}$} & \multicolumn{2}{|c|}{ Mean } & \multirow{2}{*}{$\begin{array}{c}\text { General } \\
\text { mean }\end{array}$} & \multicolumn{2}{|c|}{300 grains $/ \mathrm{m}^{2}$} & \multicolumn{2}{|c|}{350 grains $/ \mathrm{m}^{2}$} & \multicolumn{2}{|c|}{ 400grains $/ \mathrm{m}^{2}$} & \multicolumn{2}{|c|}{ Mean } & \multirow{2}{*}{$\begin{array}{c}\text { General } \\
\text { mean }\end{array}$} \\
\hline & & $75 \mathrm{KgN}$ & $90 \mathrm{KgN}$ & $75 \mathrm{KgN}$ & $90 \mathrm{KgN}$ & $75 \mathrm{KgN}$ & $90 \mathrm{KgN}$ & $75 \mathrm{KgN}$ & $90 \mathrm{KgN}$ & & $75 \mathrm{KgN}$ & $90 \mathrm{KgN}$ & $75 \mathrm{KgN}$ & $90 \mathrm{KgN}$ & $75 \mathrm{KgN}$ & $90 \mathrm{KgN}$ & $75 \mathrm{KgN}$ & $90 \mathrm{KgN}$ & & $75 \mathrm{KgN}$ & $90 \mathrm{KgN}$ & $75 \mathrm{KgN}$ & $90 \mathrm{KgN}$ & $75 \mathrm{KgN}$ & $90 \mathrm{KgN}$ & $75 \mathrm{KgN}$ & $90 \mathrm{KgN}$ & \\
\hline Sakha 93 & & \begin{tabular}{|l|}
94.33 \\
\end{tabular} & 98.33 & 90.67 & 91.67 & 85.67 & 88.33 & 90.22 & \begin{tabular}{|l|}
92.78 \\
\end{tabular} & $91.50 \mathrm{c}$ & 93.33 & 94.67 & 90.00 & 90.00 & 85.67 & 83.33 & \begin{tabular}{|l|}
89.67 \\
\end{tabular} & \begin{tabular}{|l|}
89.33 \\
\end{tabular} & $89.50 \mathrm{c}$ & \begin{tabular}{|l|}
93.83 \\
\end{tabular} & 96.50 & 90.33 & 90.83 & 85.67 & 85.83 & 89.94 & 91.06 & $90.50 c$ \\
\hline Sakha 94 & 莺 & 103.33 & 108.33 & 95.00 & \begin{tabular}{|l|}
95.67 \\
\end{tabular} & \begin{tabular}{|l|}
90.00 \\
\end{tabular} & \begin{tabular}{|l|}
91.67 \\
\end{tabular} & 96.11 & \begin{tabular}{|l|}
98.56 \\
\end{tabular} & $97.53 \mathrm{~b}$ & 98.33 & 98.33 & 96.67 & \begin{tabular}{|l|}
98.33 \\
\end{tabular} & 90.00 & 88.33 & \begin{tabular}{|l|}
95.00 \\
\end{tabular} & \begin{tabular}{|l|}
95.00 \\
\end{tabular} & $95.00 \mathrm{~b}$ & 100.83 & 103.33 & 95.83 & 97.00 & 90.00 & \begin{tabular}{|l|}
90.00 \\
\end{tabular} & 95.56 & 96.78 & $96.17 \mathrm{~b}$ \\
\hline Gemmeiza10 & 造 & 91.67 & 96.67 & 89.00 & 96.33 & 85.00 & 83.33 & 88.56 & \begin{tabular}{|l|}
92.11 \\
\end{tabular} & $90.33 c$ & 91.67 & 91.67 & 87.33 & 88.33 & 84.00 & 86.33 & \begin{tabular}{|l|}
87.67 \\
\end{tabular} & \begin{tabular}{|l|}
88.78 \\
\end{tabular} & $88.22 \mathrm{c}$ & 91.67 & \begin{tabular}{|l|}
94.17 \\
\end{tabular} & 88.17 & 92.33 & 84.50 & 84.83 & 88.11 & 90.44 & $89.28 \mathrm{c}$ \\
\hline Sids 12 & & 106.67 & 110.00 & 93.33 & 100.00 & 93.33 & 91.67 & 97.78 & 100.56 & $99.17 \mathrm{~b}$ & 100.00 & 103.33 & 93.33 & \begin{tabular}{|l|}
98.33 \\
\end{tabular} & 88.33 & 91.67 & \begin{tabular}{|l|l|}
93.89 \\
\end{tabular} & \begin{tabular}{|l|l|}
97.78 \\
\end{tabular} & $95.83 \mathrm{~b}$ & 103.33 & 106.67 & 93.33 & 99.17 & 90.83 & 91.67 & 95.83 & 99.17 & $97.50 \mathrm{~b}$ \\
\hline \begin{tabular}{|l|} 
Beni- \\
Sweif 4
\end{tabular} & 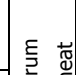 & 103.33 & 110.00 & \begin{tabular}{|c|}
98.33 \\
\end{tabular} & 103.33 & \begin{tabular}{|l|}
90.00 \\
\end{tabular} & 95.00 & 97.22 & 102.78 & $100.00 a b$ & 101.67 & 108.33 & 98.33 & |106.67| & 90.00 & 93.33 & \begin{tabular}{|l|}
96.67 \\
\end{tabular} & 102.78 & 99.72a & 102.50 & $\mid 109.17$ & 98.33 & 105.00 & 90.00 & 94.17 & 96.94 & 102.78 & 99.86a \\
\hline \begin{tabular}{|l} 
Beni- \\
Sweif 5
\end{tabular} & $\begin{array}{ll}\overline{0} & \xi \\
\end{array}$ & 106.67 & 111.67 & 100.00 & 105.00 & \begin{tabular}{|l|}
91.67 \\
\end{tabular} & $\mid 96.67$ & 99.44 & \begin{tabular}{|l|l|}
104.44 \\
\end{tabular} & $101.94 a$ & 105.00 & 106.67 & 100.00 & 105.00 & 90.00 & $\mid 96.00$ & \begin{tabular}{|l|}
98.33 \\
\end{tabular} & 102.56 & $100.44 a$ & 105.83 & $\mid 109.17$ & 100.00 & 105.00 & | 90.83 & \begin{tabular}{|l|}
96.33 \\
\end{tabular} & 98.89 & 103.50 & 101.19a \\
\hline Mean & & 101.00 & 105.83 & 94.39 & 98.67 & \begin{tabular}{|l|}
89.28 \\
\end{tabular} & \begin{tabular}{|l|l}
91.11 \\
\end{tabular} & $94.89 \mathrm{~b}$ & 98.54a & - & \begin{tabular}{|l|}
98.33 \\
\end{tabular} & 100.50 & \begin{tabular}{|l|}
94.28 \\
\end{tabular} & 97.78 & 88.00 & 89.83 & $93.54 \mathrm{~b}$ & 96.04a & - & \begin{tabular}{|l|}
99.67 \\
\end{tabular} & 103.17 & 94.33 & \begin{tabular}{|l|}
98.22 \\
\end{tabular} & 88.64 & \begin{tabular}{|l|}
90.47 \\
\end{tabular} & $94.21 \mathrm{~b}$ & $97.29 \mathrm{a}$ & - \\
\hline General mea & & 103. & $42 a$ & 96.5 & & 90.1 & & & - & & 99.4 & & 96. & & 88.9 & $.92 c$ & & - & & 101. & $1.42 \mathrm{a}$ & 96.2 & $.28 \mathrm{~b}$ & 89.5 & $56 \mathrm{c}$ & & - & \\
\hline Interaction & & & & & & N.S. & & & & & & & & & N.S. & & & & & & & & & N.S. & & & & \\
\hline & & & & & & & & & & & & & No. of & f spikes & $/ \mathrm{m}^{2}$ & & & & & & & & & & & & & \\
\hline Sakha 93 & & 477.00 & 484.67 & 487.33 & 490.67 & 495.00 & 504.00 & \begin{tabular}{|l|l|}
486.44 \\
\end{tabular} & 493.33 & 489.84a & 490.67 & 491.00 & 498.67 & 492.00 & 495.00 & 519.00 & \begin{tabular}{|l|l|}
494.11 \\
\end{tabular} & \begin{tabular}{|l|}
500.67 \\
\end{tabular} & $497.39 \mathrm{~b}$ & 483.83 & 487.83 & 493.00 & \begin{tabular}{|l|}
491.33 \\
\end{tabular} & 494.00 & 511.83 & 490.28 & 497.00 & $493.44 c$ \\
\hline Sakha 94 & ర్లుర & 488.33 & 486.33 & \begin{tabular}{|l|l|}
497.67 \\
\end{tabular} & 502.33 & 513.33 & 523.00 & \begin{tabular}{|l|l|}
499.78 \\
\end{tabular} & \begin{tabular}{|l|l|}
503.89 \\
\end{tabular} & 501.83a & 514.00 & 498.67 & 499.67 & 497.33 & 537.00 & 544.33 & 516.89 & \begin{tabular}{|l|l|}
513.44 \\
\end{tabular} & $515.17 \mathrm{a}$ & 501.17 & 492.50 & 498.67 & \begin{tabular}{|l|l|}
499.83 \\
\end{tabular} & 525.17 & 533.67 & 508.33 & 508.67 & $508.50 \mathrm{ab}$ \\
\hline Gemmeiza10 & 造 & 476.67 & 484.33 & 481.00 & 503.67 & 487.00 & 519.33 & 481.56 & \begin{tabular}{|l|l|}
502.44 \\
\end{tabular} & $492.00 \mathrm{a}$ & 486.67 & 499.00 & 484.67 & 495.67 & 498.00 & 508.00 & \begin{tabular}{|l|l|}
489.78 \\
\end{tabular} & 500.89 & $495.33 \mathrm{~b}$ & 481.67 & 491.67 & 482.83 & \begin{tabular}{|l|}
499.67 \\
\end{tabular} & 492.50 & 513.67 & 485.67 & 501.67 & $493.67 c$ \\
\hline Sids 12 & & 479.67 & 492.67 & 486.33 & 520.00 & 527.33 & 540.00 & 497.78 & \begin{tabular}{|l|l|}
517.67 \\
\end{tabular} & $07.72 a$ & 507.00 & 504.33 & 517.33 & 512.67 & 551.67 & 553.33 & 525.33 & 523.44 & 524.39a & 493.33 & 498.50 & 501.83 & 516.33 & 539.50 & 546.83 & 511.56 & 520.56 & $516.06 a$ \\
\hline \begin{tabular}{|l}
$\begin{array}{l}\text { Beni- } \\
\text { Sweif } 4\end{array}$ \\
\end{tabular} & E & 479.00 & |496.00 & 480.33 & 515.33 & 505.00 & 536.00 & \begin{tabular}{|l|l|} 
& 488.11 \\
\end{tabular} & 5.78 & $.94 a$ & 1.67 & 517.67 & 475.67 & .67 & \begin{tabular}{|l|l|}
489.00 \\
\end{tabular} & 543.00 & \begin{tabular}{|l|l|}
479.78 \\
\end{tabular} & 529.44 & $4.61 \mathrm{~b}$ & 476.83 & 506.83 & 478.00 & 521.50 & 457.00 & 539.50 & 483.94 & 522.61 & $503.28 \mathrm{bc}$ \\
\hline $\begin{array}{l}\text { Beni- } \\
\text { Sweif } 5\end{array}$ & & 485.67 & 522.33 & |486.67| & 526.33 & 511.00 & 556.00 & $\mid 494.44$ & 534.89 & 514.67a & 492.33 & 534.00 & 496.00 & 536.00 & 523.00 & 552.00 & $\mid 503.89$ & 540.67 & $522.28 \mathrm{a}$ & 489.00 & 528.17 & 491.33 & |531.17 & 517.17 & 554.00 & 499.17 & 537.78 & 518.47a \\
\hline Mean & & 481.06 & 494.39 & 486.56 & 509.72 & 505.44 & 529.89 & $491.35 \mathrm{~b}$ & $511.33 \mathrm{a}$ & - & 494.22 & 507.44 & 495.33 & 510.22 & 515.33 & 536.61 & $501.63 \mathrm{~b}$ & $518.09 \mathrm{a}$ & - & 487.64 & 500.92 & 490.94 & 509.97 & 510.89 & 533.25 & $496.49 \mathrm{~b}$ & $514.71 \mathrm{a}$ & - \\
\hline General mea & & 487. & & 498. & & 518. & & & - & & 500. & & 502. & & 525. & & & - & & & & 500. & $46 \mathrm{~b}$ & & & & - & \\
\hline Interaction & & & & & & N.S. & & & & & & & & & N.S. & & & & & & & & & N.S & & & & \\
\hline
\end{tabular}

Means followed by the same letter (s) in each column within each treatment are not significant. 
Table 3. Effect of seeding and nitrogen fertilizer rates on number of grains/spike and 1000-grain weight of some wheat cultivars during the two successive seasons (2009/10 and 2010/11).

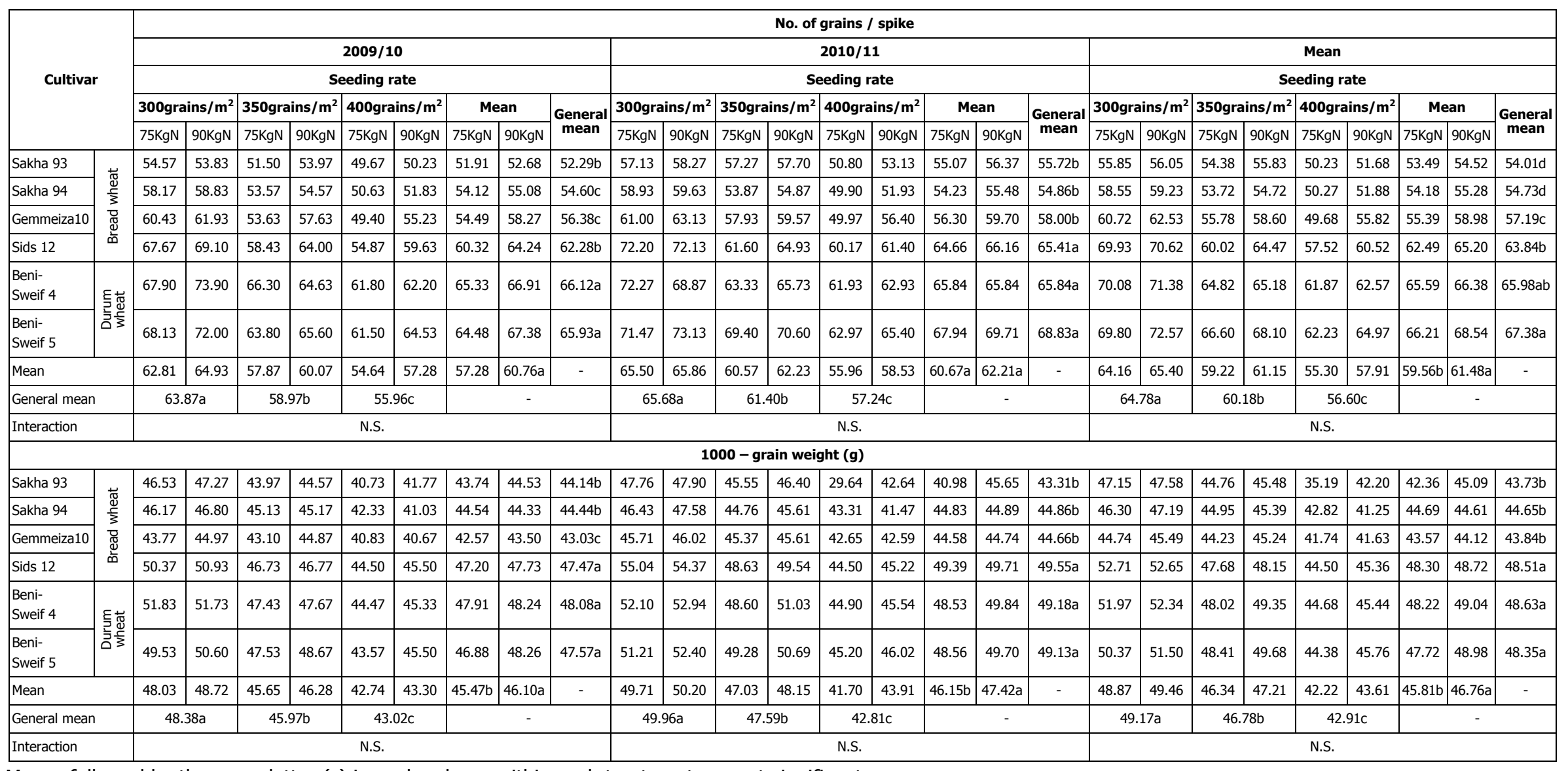

Means followed by the same letter (s) in each column within each treatment are not significant. 
Table 4. Effect of seeding and nitrogen fertilizer rates on grain and straw yields of some wheat cultivars during the two successive seasons (2009/10 and 2010/11).

\begin{tabular}{|c|c|c|c|c|c|c|c|c|c|c|c|c|c|c|c|c|c|c|c|c|c|c|c|c|c|c|c|c|}
\hline \multirow{5}{*}{\multicolumn{2}{|c|}{ Cultivar }} & \multicolumn{27}{|c|}{ Grain yield (ardab / fed.)* } \\
\hline & & \multicolumn{9}{|c|}{ 2009/10 } & \multicolumn{9}{|c|}{$2010 / 11$} & \multicolumn{9}{|c|}{ Mean } \\
\hline & & \multicolumn{9}{|c|}{ Seeding rate } & \multicolumn{9}{|c|}{ Seeding rate } & \multicolumn{9}{|c|}{ Seeding rate } \\
\hline & & \multicolumn{2}{|c|}{ 300grains $/ \mathrm{m}^{2}$} & \multicolumn{2}{|c|}{ 350grains $/ \mathrm{m}^{2}$} & \multicolumn{2}{|c|}{ 400grains/m ${ }^{2}$} & \multicolumn{2}{|c|}{ Mean } & \multirow{2}{*}{$\begin{array}{c}\text { General } \\
\text { mean }\end{array}$} & \multicolumn{2}{|c|}{300 grains $/ \mathrm{m}^{2}$} & \multirow{2}{*}{\multicolumn{2}{|c|}{\begin{tabular}{|l|l|}
\multicolumn{3}{|c|}{ 350grains $/ \mathbf{m}^{2}$} \\
$75 \mathrm{KgN}$ & $90 \mathrm{KaN}$
\end{tabular}}} & \multicolumn{2}{|c|}{ 400grains/m ${ }^{2}$} & \multicolumn{2}{|c|}{ Mean } & \multirow{2}{*}{$\mid \begin{array}{c}\text { General } \\
\text { mean }\end{array}$} & \multicolumn{2}{|c|}{ 300grains $/ \mathrm{m}^{2}$} & \multicolumn{2}{|c|}{350 grains $/ \mathrm{m}^{2}$} & \multicolumn{2}{|c|}{400 grains $/ \mathrm{m}^{2}$} & \multicolumn{2}{|c|}{ Mean } & \multirow{2}{*}{$\begin{array}{c}\text { General } \\
\text { mean }\end{array}$} \\
\hline & & \begin{tabular}{|l|}
$75 \mathrm{KgN}$ \\
\end{tabular} & $90 \mathrm{KgN}$ & $75 \mathrm{KgN}$ & $90 \mathrm{KgN}$ & $75 \mathrm{KgN}$ & $90 \mathrm{KgN}$ & $75 \mathrm{KgN}$ & $90 \mathrm{KgN}$ & & $75 \mathrm{KgN}$ & $90 \mathrm{KgN}$ & & & $75 \mathrm{KgN}$ & $90 \mathrm{KgN}$ & $75 \mathrm{KgN}$ & $90 \mathrm{KgN}$ & & \begin{tabular}{|l|}
$75 \mathrm{KgN}$ \\
\end{tabular} & $90 \mathrm{KgN}$ & $75 \mathrm{kgN}$ & $90 \mathrm{KgN}$ & $75 \mathrm{KgN}$ & $90 \mathrm{KgN}$ & $75 \mathrm{KgN}$ & $90 \mathrm{KgN}$ & \\
\hline Sakha 93 & & \begin{tabular}{|l|}
14.87 \\
\end{tabular} & 15.47 & \begin{tabular}{|l|}
14.70 \\
\end{tabular} & 15.00 & 14.43 & \begin{tabular}{|l|}
14.67 \\
\end{tabular} & $\mid 14.67$ & 15.04 & $14.86 \mathrm{c}$ & \begin{tabular}{|l|}
15.87 \\
\end{tabular} & 16.80 & \begin{tabular}{|l|l}
15.76 & -10
\end{tabular} & 16.77 & 15.40 & \begin{tabular}{|l|}
16.34 \\
\end{tabular} & \begin{tabular}{|l|l|}
15.68 \\
\end{tabular} & 16.64 & $\mid 16.16 \mathrm{~b}$ & \begin{tabular}{|c|}
15.37 \\
\end{tabular} & 16.13 & 15.23 & 15.88 & 14.91 & 15.51 & \begin{tabular}{|l|}
15.17 \\
\end{tabular} & 15.84 & $15.51 \mathrm{c}$ \\
\hline \begin{tabular}{|l|} 
Sakha 94 \\
\end{tabular} & $\frac{\varpi}{3}$ & 14.83 & 15.53 & \begin{tabular}{|l|}
14.60 \\
\end{tabular} & 15.43 & \begin{tabular}{|l|}
14.63 \\
\end{tabular} & \begin{tabular}{|l|}
15.27 \\
\end{tabular} & 14.69 & 15.41 & $15.05 c$ & \begin{tabular}{|l|}
15.75 \\
\end{tabular} & \begin{tabular}{|l|}
17.30 \\
\end{tabular} & \begin{tabular}{|l|}
15.55 \\
\end{tabular} & \begin{tabular}{|c|}
17.07 \\
\end{tabular} & \begin{tabular}{|l|}
15.53 \\
\end{tabular} & 16.50 & \begin{tabular}{|l|}
15.61 \\
\end{tabular} & \begin{tabular}{|l|}
16.96 \\
\end{tabular} & $\mid 16.28 \mathrm{~b}$ & \begin{tabular}{|l|}
15.29 \\
\end{tabular} & 16.42 & 15.08 & 16.25 & 15.08 & \begin{tabular}{|l|}
15.88 \\
\end{tabular} & 15.15 & \begin{tabular}{|l|}
16.18 \\
\end{tabular} & $15.66 \mathrm{bc}$ \\
\hline Gemmeiza10 & $\bar{\pi}_{\pi}^{\circ}$ & 15.01 & 15.67 & \begin{tabular}{|l|}
14.83 \\
\end{tabular} & 15.33 & \begin{tabular}{|l|}
14.77 \\
\end{tabular} & \begin{tabular}{|l|}
15.17 \\
\end{tabular} & 14.90 & 15.39 & $15.14 \mathrm{bc}$ & \begin{tabular}{|l|}
16.13 \\
\end{tabular} & \begin{tabular}{|l|}
16.58 \\
\end{tabular} & 15.80 & 16.47 & \begin{tabular}{|l|}
15.37 \\
\end{tabular} & \begin{tabular}{|l|}
16.20 \\
\end{tabular} & \begin{tabular}{|l|}
15.77 \\
\end{tabular} & \begin{tabular}{|l|}
16.42 \\
\end{tabular} & $16.09 \mathrm{~b}$ & \begin{tabular}{|l|}
15.57 \\
\end{tabular} & 16.13 & 15.32 & 15.90 & 15.07 & \begin{tabular}{|l|}
15.68 \\
\end{tabular} & \begin{tabular}{|l|}
15.32 \\
\end{tabular} & 15.90 & $15.61 \mathrm{bc}$ \\
\hline Sids 12 & 产 & 16.03 & 16.50 & 15.43 & 16.00 & \begin{tabular}{|l|}
15.17 \\
\end{tabular} & \begin{tabular}{|l|}
15.77 \\
\end{tabular} & 15.54 & 16.09 & $15.82 \mathrm{a}$ & \begin{tabular}{|l|}
16.70 \\
\end{tabular} & \begin{tabular}{|l|}
17.10 \\
\end{tabular} & \begin{tabular}{|l|}
16.67 \\
\end{tabular} & 17.00 & \begin{tabular}{|l|}
16.43 \\
\end{tabular} & \begin{tabular}{|l|}
16.40 \\
\end{tabular} & \begin{tabular}{|l|}
16.60 \\
\end{tabular} & \begin{tabular}{|l|}
16.83 \\
\end{tabular} & \begin{tabular}{|l|}
$16.71 a$ \\
\end{tabular} & \begin{tabular}{|l|}
16.37 \\
\end{tabular} & 16.80 & 16.05 & 16.50 & 15.80 & \begin{tabular}{|l|}
16.08 \\
\end{tabular} & \begin{tabular}{|l|}
16.07 \\
\end{tabular} & \begin{tabular}{|l|}
16.46 \\
\end{tabular} & $16.26 a$ \\
\hline $\begin{array}{l}\text { Beni- } \\
\text { Sweif } 4\end{array}$ & \pm & \begin{tabular}{|l|}
15.93 \\
\end{tabular} & 16.83 & \begin{tabular}{|l|}
15.63 \\
\end{tabular} & 15.83 & 15.50 & 15.70 & 15.69 & 16.12 & 15.91a & $\mid 16.57$ & 17.73 & 16.50 & 16.73 & 15.80 & $\mid 15.90$ & 16.29 & 16.79 & 16.54ab & 16.25 & 17.28 & 16.06 & 16.28 & 15.65 & 15.80 & 15.99 & 16.45 & $16.22 \mathrm{a}$ \\
\hline \begin{tabular}{|l} 
Beni- \\
Sweif 5
\end{tabular} & & \begin{tabular}{|l|}
15.53 \\
\end{tabular} & 16.17 & $\mid 14.93$ & 16.00 & 14.80 & 15.70 & 15.09 & 15.96 & $15.52 \mathrm{ab}$ & $\mid 16.17$ & $\mid 17.27$ & 15.77 & 16.20 & 15.17 & $\mid 16.07$ & 15.70 & 16.51 & $16.11 \mathrm{~b}$ & 15.85 & 16.72 & 15.35 & 16.11 & 14.98 & 15.88 & 15.39 & 16.24 & $15.81 \mathrm{~b}$ \\
\hline Mean & & 15.37 & 16.03 & \begin{tabular}{|l|}
15.02 \\
\end{tabular} & 15.60 & \begin{tabular}{|l|l|}
14.88 & \\
\end{tabular} & \begin{tabular}{|l|l|}
15.38 \\
\end{tabular} & $15.10 \mathrm{~b}$ & $15.67 a$ & - & $\mid$\begin{tabular}{|l|}
16.20 \\
\end{tabular} & \begin{tabular}{|l|}
17.13 \\
\end{tabular} & \begin{tabular}{|l|l|}
16.01 &
\end{tabular} & 16.71 & 15.62 & \begin{tabular}{|l|}
16.23 \\
\end{tabular} & $15.94 \mathrm{~b}$ & 16.69a & & \begin{tabular}{|l|}
15.79 \\
\end{tabular} & 16.58 & 15.51 & 16.15 & 15.25 & 15.81 & $15.52 \mathrm{~b}$ & $16.18 \mathrm{a}$ & \\
\hline General mean & & 15.7 & $70 \mathrm{a}$ & 15.3 & & 15. & $13 \mathrm{c}$ & & - & & 16.6 & & 16.3 & & 15. & $92 \mathrm{c}$ & & - & & 16.1 & & 15.8 & $33 \mathrm{~b}$ & 15. & $53 \mathrm{c}$ & & - & \\
\hline Interaction & & & & & & N.S. & & & & & & & & & N.S. & & & & & & & & & N.S. & & & & \\
\hline & & & & & & & & & & & & & raw yiel & ld (ton/ & fed.)* & & & & & & & & & & & & & \\
\hline Sakha 93 & + & 2.96 & 3.28 & 3.11 & 3.32 & 3.27 & 3.43 & 3.11 & 3.34 & $3.23 a$ & 3.14 & 3.35 & 3.24 & 3.35 & 3.39 & 3.35 & 3.26 & 3.35 & $3.30 \mathrm{a}$ & 3.05 & 3.32 & 3.17 & 3.34 & 3.33 & \begin{tabular}{|l|}
3.39 \\
\end{tabular} & 3.25 & 3.18 & $3.36 \mathrm{c}$ \\
\hline Sakha 94 & $\frac{\mathbb{d}}{3}$ & 3.41 & 3.32 & 3.39 & 3.49 & 3.60 & \begin{tabular}{|l|}
3.51 \\
\end{tabular} & 3.47 & 3.44 & $3.45 a$ & 3.23 & 3.33 & \begin{tabular}{|l|}
3.30 \\
\end{tabular} & 3.41 & 3.54 & 3.41 & 3.36 & 3.38 & $3.37 a$ & 3.32 & 3.32 & 3.35 & 3.45 & 3.57 & 3.46 & 3.40 & 3.32 & $3.51 \mathrm{ab}$ \\
\hline Gemmeiza10 & $\frac{1}{\pi}$ & 3.04 & 3.10 & 3.25 & 3.31 & 3.33 & \begin{tabular}{|l|}
3.56 \\
\end{tabular} & 3.21 & 3.32 & $3.26 \mathrm{a}$ & 3.30 & 3.35 & 3.20 & 3.39 & 3.48 & 3.51 & \begin{tabular}{|l|}
3.33 \\
\end{tabular} & 3.42 & $3.37 a$ & 3.17 & 3.23 & 3.22 & 3.35 & 3.40 & 3.53 & 3.29 & 3.20 & $3.47 \mathrm{~b}$ \\
\hline Sids 12 & 产 & 3.09 & 3.32 & 3.32 & 3.52 & 3.39 & \begin{tabular}{|l|}
3.58 \\
\end{tabular} & 3.27 & 3.47 & $3.37 a$ & 3.30 & 3.45 & 3.26 & 3.45 & 3.38 & 3.50 & 3.32 & 3.47 & $3.39 a$ & 3.20 & 3.38 & 3.29 & 3.48 & 3.39 & \begin{tabular}{|l|}
3.54 \\
\end{tabular} & 3.39 & 3.29 & $3.46 \mathrm{bc}$ \\
\hline \begin{tabular}{|l} 
Beni- \\
Sweif 4
\end{tabular} & 严 苋 & 3.34 & 3.39 & 3.47 & 3.51 & 3.64 & 3.69 & 3.48 & 3.53 & 3.51a & 3.30 & \begin{tabular}{|l|l} 
& 3.47 \\
\end{tabular} & 3.35 & 3.49 & 3.46 & 3.53 & 3.37 & 3.49 & 3.43a & 3.32 & 3.43 & 3.41 & 3.50 & 3.55 & 3.61 & 3.45 & 3.37 & $3.58 \mathrm{a}$ \\
\hline $\begin{array}{l}\text { Beni- } \\
\text { Sweif } 5\end{array}$ & & 3.20 & 3.23 & 3.47 & 3.44 & 3.49 & 3.48 & 3.38 & 3.38 & $3.38 a$ & 3.29 & 3.46 & 3.42 & 3.53 & 3.51 & 3.52 & 3.41 & 3.50 & $3.45 a$ & 3.25 & 3.34 & 3.44 & 3.48 & 3.50 & 3.50 & 3.46 & 3.29 & 3.50ab \\
\hline Mean & & 3.17 & 3.27 & 3.34 & 3.43 & 3.45 & 3.54 & $3.32 \mathrm{~b}$ & $3.42 \mathrm{a}$ & - & 3.26 & 3.40 & 3.29 & 3.44 & 3.46 & 3.47 & $3.34 \mathrm{~b}$ & $3.43 a$ & - & 3.22 & 3.33 & 3.38 & 3.36 & 3.50 & 3.46 & 3.33b & $3.42 a$ & - \\
\hline General mean & & 3.2 & $2 c$ & 3.38 & $38 \mathrm{~b}$ & & $50 \mathrm{a}$ & & & & 3.3 & & 3.36 & & 3.4 & $46 a$ & & - & & 3.2 & & 3.3 & $7 \mathrm{~b}$ & 3.4 & $8 a$ & & & \\
\hline Interaction & & & & & & N.S. & & & & & & & & & N.S. & & & & & & & & & N.S. & & & & \\
\hline
\end{tabular}

Means followed by the same letter (s) in each column within each treatment are not significant.

$*$ Ardab $=150 \mathrm{~kg}$. Ton $=1000 \mathrm{Kg}$. Fed. $=4200 \mathrm{~m}^{2}$ 
Table 5. Effect of seeding and nitrogen fertilizer rates on grain crude of protein and total carbohydrate percentages of some wheat cultivars during the two successive seasons (2009/10 and 2010/11).

\begin{tabular}{|c|c|c|c|c|c|c|c|c|c|c|c|c|c|c|c|c|c|c|c|c|c|c|c|c|c|c|c|c|}
\hline \multirow{5}{*}{\multicolumn{2}{|c|}{ Cultivar }} & \multicolumn{27}{|c|}{ Protein \% } \\
\hline & & \multicolumn{9}{|c|}{$2009 / 10$} & \multicolumn{9}{|c|}{ 2010/11 } & \multicolumn{9}{|c|}{ Mean } \\
\hline & & \multicolumn{9}{|c|}{ Seeding rate } & \multicolumn{9}{|c|}{ Seeding rate } & \multicolumn{9}{|c|}{ Seeding rate } \\
\hline & & \multicolumn{2}{|c|}{ 300grains/m² } & \multirow{2}{*}{\multicolumn{2}{|c|}{ 350grains $/ \mathrm{m}^{2}$}} & \multicolumn{2}{|c|}{400 grains $/ \mathrm{m}^{2}$} & \multicolumn{2}{|c|}{ Mean } & \multirow{2}{*}{$\begin{array}{c}\text { General } \\
\text { mean }\end{array}$} & \multicolumn{2}{|c|}{ 300grains $/ \mathbf{m}^{2}$} & \multicolumn{2}{|c|}{ 350grains $/ \mathbf{m}^{2}$} & \multicolumn{2}{|c|}{ 400grains $/ \mathrm{m}^{2}$} & \multicolumn{2}{|c|}{ Mean } & \multirow{2}{*}{$\begin{array}{c}\text { General } \\
\text { mean }\end{array}$} & \multicolumn{2}{|c|}{ 300grains $/ \mathrm{m}^{2}$} & \multicolumn{2}{|c|}{ 350grains $/ \mathrm{m}^{2}$} & \multicolumn{2}{|c|}{ 400grains $/ \mathrm{m}^{2}$} & \multicolumn{2}{|c|}{ Mean } & \multirow{2}{*}{$\begin{array}{c}\text { General } \\
\text { mean }\end{array}$} \\
\hline & & $75 \mathrm{KgN}$ & $90 \mathrm{KgN}$ & $75 \mathrm{KgN}$ & & $75 \mathrm{kgN}$ & $90 \mathrm{KgN}$ & $75 \mathrm{KgN}$ & $90 \mathrm{KgN}$ & & $75 \mathrm{KgN}$ & $90 \mathrm{KgN}$ & $75 \mathrm{KgN}$ & $90 \mathrm{KgN}$ & $75 \mathrm{KgN}$ & $90 \mathrm{KgN}$ & $75 \mathrm{KgN}$ & $90 \mathrm{KgN}$ & & 75KgN & $90 \mathrm{KgN}$ & \begin{tabular}{|l|l|}
$75 \mathrm{KgN}$ \\
\end{tabular} & $90 \mathrm{KgN}$ & $75 \mathrm{KgN}$ & $90 \mathrm{KgN}$ & $75 \mathrm{KgN}$ & $90 \mathrm{KgN}$ & \\
\hline \begin{tabular}{|l|} 
Sakha 93 \\
\end{tabular} & & \begin{tabular}{|l|}
10.47 \\
\end{tabular} & \begin{tabular}{|l|}
10.67 \\
\end{tabular} & \begin{tabular}{|l|}
10.27 \\
\end{tabular} & 10.50 & 10.13 & \begin{tabular}{|l|}
10.30 \\
\end{tabular} & 10.29 & 10.49 & $\begin{array}{ll}10.39 d \\
\end{array}$ & \begin{tabular}{|l|}
10.52 \\
\end{tabular} & \begin{tabular}{|l|}
10.67 \\
\end{tabular} & \begin{tabular}{|l|}
10.46 \\
\end{tabular} & 10.53 & 10.36 & \begin{tabular}{|l|}
10.43 \\
\end{tabular} & \begin{tabular}{|l|}
10.44 \\
\end{tabular} & 10.55 & $\begin{array}{ll}10.49 d \\
\end{array}$ & \begin{tabular}{|l|}
10.49 \\
\end{tabular} & \begin{tabular}{|l|}
10.67 \\
\end{tabular} & \begin{tabular}{|l|}
10.36 \\
\end{tabular} & 10.52 & \begin{tabular}{|l|}
10.25 \\
\end{tabular} & 10.37 & \begin{tabular}{|l|}
10.37 \\
\end{tabular} & \begin{tabular}{|l|}
10.52 \\
\end{tabular} & $10.44 \mathrm{e}$ \\
\hline Sakha 94 & 范 & \begin{tabular}{|l|}
11.80 \\
\end{tabular} & \begin{tabular}{|l|}
11.67 \\
\end{tabular} & \begin{tabular}{|l|}
11.27 \\
\end{tabular} & \begin{tabular}{|l|}
11.47 \\
\end{tabular} & 10.67 & \begin{tabular}{|l|}
10.63 \\
\end{tabular} & 11.24 & 11.26 & $11.25 \mathrm{c}$ & \begin{tabular}{|l|}
12.12 \\
\end{tabular} & \begin{tabular}{|l|}
12.19 \\
\end{tabular} & \begin{tabular}{|l|}
11.99 \\
\end{tabular} & 11.88 & 11.32 & \begin{tabular}{|l|}
11.32 \\
\end{tabular} & \begin{tabular}{|l|}
11.81 \\
\end{tabular} & \begin{tabular}{|l|}
11.80 \\
\end{tabular} & $11.80 \mathrm{~b}$ & \begin{tabular}{|l|}
11.96 \\
\end{tabular} & \begin{tabular}{|l|}
11.93 \\
\end{tabular} & $\mid 11.63$ & 11.68 & \begin{tabular}{|l|}
10.99 \\
\end{tabular} & 10.98 & 11.53 & \begin{tabular}{|l|}
11.53 \\
\end{tabular} & $11.53 \mathrm{c}$ \\
\hline Gemmeiza10 & 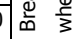 & \begin{tabular}{|l|}
12.17 \\
\end{tabular} & \begin{tabular}{|l|}
12.20 \\
\end{tabular} & \begin{tabular}{|l|}
11.83 \\
\end{tabular} & \begin{tabular}{|l|l}
11.90 \\
\end{tabular} & 10.87 & \begin{tabular}{|l|}
10.83 \\
\end{tabular} & 11.62 & 11.64 & 11.63b & \begin{tabular}{|l|}
12.08 \\
\end{tabular} & \begin{tabular}{|l|}
12.43 \\
\end{tabular} & 10.50 & \begin{tabular}{|l|}
12.10 \\
\end{tabular} & 11.65 & \begin{tabular}{|l|}
11.68 \\
\end{tabular} & \begin{tabular}{|l|}
11.41 \\
\end{tabular} & \begin{tabular}{|l|}
12.07 \\
\end{tabular} & 11.74b & \begin{tabular}{|l|}
12.12 \\
\end{tabular} & \begin{tabular}{|l|}
12.31 \\
\end{tabular} & \begin{tabular}{|l|l|}
11.17 \\
\end{tabular} & 12.00 & \begin{tabular}{|l|}
11.26 \\
\end{tabular} & 11.26 & 11.52 & 11.86 & $11.69 \mathrm{c}$ \\
\hline Sids 12 & & \begin{tabular}{|l|}
11.00 \\
\end{tabular} & \begin{tabular}{|l|}
11.07 \\
\end{tabular} & \begin{tabular}{|l|}
10.53 \\
\end{tabular} & \begin{tabular}{|l}
10.57 \\
\end{tabular} & 10.03 & \begin{tabular}{|l|}
10.17 \\
\end{tabular} & 10.52 & \begin{tabular}{|l|}
10.60 \\
\end{tabular} & $10.56 \mathrm{~d}$ & \begin{tabular}{|l|}
10.87 \\
\end{tabular} & \begin{tabular}{|l|l|}
11.17 \\
\end{tabular} & \begin{tabular}{|l|}
12.02 \\
\end{tabular} & \begin{tabular}{|l|}
10.73 \\
\end{tabular} & 10.27 & \begin{tabular}{|l|}
10.31 \\
\end{tabular} & \begin{tabular}{|l|}
11.05 \\
\end{tabular} & 10.74 & $10.89 \mathrm{c}$ & \begin{tabular}{|l|}
10.93 \\
\end{tabular} & \begin{tabular}{|l|}
11.12 \\
\end{tabular} & \begin{tabular}{|l|}
11.27 \\
\end{tabular} & 10.65 & \begin{tabular}{|l|}
10.15 \\
\end{tabular} & 10.24 & \begin{tabular}{|l|}
10.79 \\
\end{tabular} & \begin{tabular}{|l|}
10.67 \\
\end{tabular} & $10.73 d$ \\
\hline \begin{tabular}{|l} 
Beni- \\
Sweif 4
\end{tabular} & E & $\mid$\begin{tabular}{|l|}
12.20 \\
\end{tabular} & \begin{tabular}{|l|}
12.17 \\
\end{tabular} & $\mid 11.83$ & 11.93 & 11.40 & $\mid 11.60$ & 11.81 & 11.90 & $11.86 a b$ & $\mid 12.40$ & 12.53 & 12.02 & $\mid 12.13$ & 11.67 & \begin{tabular}{|l|l|}
11.57 \\
\end{tabular} & \begin{tabular}{|l|}
12.03 \\
\end{tabular} & 12.08 & $12.05 \mathrm{ab}$ & \begin{tabular}{|l|}
12.30 \\
\end{tabular} & 12.35 & \begin{tabular}{|l|}
11.92 \\
\end{tabular} & 12.03 & $\mid 11.53$ & 11.58 & 11.92 & 11.99 & $11.95 \mathrm{~b}$ \\
\hline \begin{tabular}{|l} 
Beni- \\
Sweif 5
\end{tabular} & $7 \overline{5} \frac{5}{2}$ & \begin{tabular}{|l|}
12.40 \\
\end{tabular} & 12.80 & 12.03 & 11.87 & 11.67 & \begin{tabular}{|l|l|}
11.87 \\
\end{tabular} & 12.03 & 12.18 & $12.11 \mathrm{a}$ & 12.79 & 12.80 & 12.10 & 12.43 & 11.84 & \begin{tabular}{|l|l|}
11.67 \\
\end{tabular} & \begin{tabular}{|l|}
12.24 \\
\end{tabular} & 12.30 & $12.27 \mathrm{a}$ & \begin{tabular}{|l|}
12.59 \\
\end{tabular} & $\mid 12.80$ & \begin{tabular}{|l|}
12.07 \\
\end{tabular} & 12.15 & 11.75 & 11.77 & 12.14 & 12.24 & $12.19 a$ \\
\hline Mean & & \begin{tabular}{|l|}
11.67 \\
\end{tabular} & \begin{tabular}{|l|l|}
11.76 \\
\end{tabular} & \begin{tabular}{|l|}
11.29 \\
\end{tabular} & \begin{tabular}{|l|l}
11.37 \\
\end{tabular} & 10.79 & \begin{tabular}{|l|}
10.90 \\
\end{tabular} & $11.25 \mathrm{~b}$ & $11.34 a$ & - & \begin{tabular}{|l|}
11.79 \\
\end{tabular} & \begin{tabular}{|l|}
11.96 \\
\end{tabular} & \begin{tabular}{|l|}
11.51 \\
\end{tabular} & \begin{tabular}{|l|}
11.64 \\
\end{tabular} & \begin{tabular}{|l|l|}
11.18 \\
\end{tabular} & \begin{tabular}{|l|}
11.16 \\
\end{tabular} & 11.50a & 11.59a & - & \begin{tabular}{|l|}
11.73 \\
\end{tabular} & \begin{tabular}{|l|l|}
11.86 \\
\end{tabular} & \begin{tabular}{|l|}
11.40 \\
\end{tabular} & 11.50 & \begin{tabular}{|l|}
10.99 \\
\end{tabular} & \begin{tabular}{|l|}
11.03 \\
\end{tabular} & $11.38 \mathrm{~b}$ & $11.47 \mathrm{a}$ & - \\
\hline General mea & & 11. & $.72 a$ & 11. & $33 \mathrm{~b}$ & 10. & & & - & & 11. & $88 \mathrm{a}$ & 11. & & 11. & $.17 \mathrm{c}$ & & - & & 11.8 & & 11.4 & $45 b$ & 11. & $.01 \mathrm{c}$ & & - & \\
\hline Interaction & & & & & & N.S. & & & & & & & & & N.S. & & & & & & & & & N.S. & & & & \\
\hline & & & & & & & & & & & & & otal carb & bohydra & $\%$ & & & & & & & & & & & & & \\
\hline Sakha 93 & & \begin{tabular}{|l|}
71.27 \\
\end{tabular} & 71.67 & \begin{tabular}{|l|}
69.50 \\
\end{tabular} & \begin{tabular}{|l|l|}
70.77 \\
\end{tabular} & 69.30 & \begin{tabular}{|l|}
70.73 \\
\end{tabular} & 70.02 & \begin{tabular}{|l|}
71.06 \\
\end{tabular} & $70.54 \mathrm{c}$ & \begin{tabular}{|l|}
71.17 \\
\end{tabular} & \begin{tabular}{|l|l|}
72.67 \\
\end{tabular} & 70.27 & \begin{tabular}{|l|l|}
71.37 \\
\end{tabular} & 68.97 & \begin{tabular}{|l|}
69.17 \\
\end{tabular} & \begin{tabular}{|l|}
70.13 \\
\end{tabular} & 71.07 & $70.60 \mathrm{~b}$ & 71.22 & \begin{tabular}{|l|}
72.17 \\
\end{tabular} & \begin{tabular}{|l|}
69.88 \\
\end{tabular} & 71.07 & \begin{tabular}{|l|}
69.13 \\
\end{tabular} & \begin{tabular}{|l|l|}
69.95 \\
\end{tabular} & \begin{tabular}{|l|}
70.08 \\
\end{tabular} & \begin{tabular}{|l|}
71.06 \\
\end{tabular} & $70.57 c$ \\
\hline Sakha 94 & 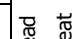 & \begin{tabular}{|l|}
71.10 \\
\end{tabular} & \begin{tabular}{|l|}
72.40 \\
\end{tabular} & \begin{tabular}{|l|}
70.17 \\
\end{tabular} & \begin{tabular}{|l|l|}
71.37 \\
\end{tabular} & 69.83 & \begin{tabular}{|l|}
70.43 \\
\end{tabular} & 70.37 & 71.40 & $70.88 \mathrm{c}$ & \begin{tabular}{|l|}
71.13 \\
\end{tabular} & \begin{tabular}{|l|}
73.40 \\
\end{tabular} & 70.27 & \begin{tabular}{|l|}
70.67 \\
\end{tabular} & 69.13 & \begin{tabular}{|l|}
69.80 \\
\end{tabular} & \begin{tabular}{|l|}
70.18 \\
\end{tabular} & 71.29 & $70.73 b$ & \begin{tabular}{|l}
71.12 \\
\end{tabular} & \begin{tabular}{|l|}
72.90 \\
\end{tabular} & \begin{tabular}{|l|}
70.22 \\
\end{tabular} & 71.02 & \begin{tabular}{|l|}
69.48 \\
\end{tabular} & \begin{tabular}{|l|}
70.12 \\
\end{tabular} & \begin{tabular}{|l|}
70.27 \\
\end{tabular} & \begin{tabular}{|l|}
71.34 \\
\end{tabular} & $70.81 \mathrm{c}$ \\
\hline Gemmeiza10 & 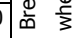 & \begin{tabular}{|l|}
69.50 \\
\end{tabular} & \begin{tabular}{|l|}
70.20 \\
\end{tabular} & \begin{tabular}{|l|l|}
68.83 \\
\end{tabular} & \begin{tabular}{|l|l}
69.93 \\
\end{tabular} & 67.40 & \begin{tabular}{|l|}
69.17 \\
\end{tabular} & \begin{tabular}{|l|}
68.58 \\
\end{tabular} & \begin{tabular}{|l|l|}
69.77 \\
\end{tabular} & $69.17 \mathrm{~d}$ & \begin{tabular}{|l|l|}
69.60 \\
\end{tabular} & \begin{tabular}{|l|}
70.63 \\
\end{tabular} & \begin{tabular}{|l|}
69.20 \\
\end{tabular} & \begin{tabular}{|l|l|}
69.50 \\
\end{tabular} & $\begin{array}{lll}68.17 \\
\end{array}$ & \begin{tabular}{|l|l|}
68.67 \\
\end{tabular} & \begin{tabular}{|l|l|}
68.99 \\
\end{tabular} & \begin{tabular}{|l|}
69.60 \\
\end{tabular} & \begin{tabular}{|l|l|}
$69.29 c$ \\
\end{tabular} & \begin{tabular}{|l|l|}
69.55 \\
\end{tabular} & \begin{tabular}{|l|l|}
70.42 \\
\end{tabular} & \begin{tabular}{|l|}
69.02 \\
\end{tabular} & 69.72 & \begin{tabular}{|l|}
67.78 \\
\end{tabular} & \begin{tabular}{|l|}
68.92 \\
\end{tabular} & \begin{tabular}{|l|l|}
68.78 \\
\end{tabular} & \begin{tabular}{|l|}
69.68 \\
\end{tabular} & $69.23 \mathrm{e}$ \\
\hline Sids 12 & & \begin{tabular}{|l|}
70.77 \\
\end{tabular} & \begin{tabular}{|l|}
72.00 \\
\end{tabular} & 69.33 & 70.57 & 68.73 & \begin{tabular}{|l|l|}
69.53 \\
\end{tabular} & \begin{tabular}{|l|}
69.61 \\
\end{tabular} & 70.70 & $70.16 \mathrm{c}$ & 70.63 & \begin{tabular}{|l|l|}
71.30 \\
\end{tabular} & 70.10 & \begin{tabular}{|l}
70.37 \\
\end{tabular} & 68.87 & \begin{tabular}{|l|l|}
69.43 \\
\end{tabular} & \begin{tabular}{|l|l|}
69.87 \\
\end{tabular} & 70.37 & $70.12 b$ & 70.70 & 71.65 & \begin{tabular}{|l|}
69.72 \\
\end{tabular} & \begin{tabular}{|l|l|}
70.47 \\
\end{tabular} & \begin{tabular}{|l|l|}
68.80 \\
\end{tabular} & \begin{tabular}{|l|}
69.48 \\
\end{tabular} & \begin{tabular}{|l|l|}
69.74 \\
\end{tabular} & 70.53 & $70.14 d$ \\
\hline \begin{tabular}{|l|} 
Beni- \\
Sweif 4 \\
\end{tabular} & E & \begin{tabular}{|l|l|} 
& 76.40 \\
\end{tabular} & 76.53 & \begin{tabular}{|l|l|}
74.57 \\
\end{tabular} & 75.87 & 74.67 & \begin{tabular}{|l|l|} 
& 74.43 \\
\end{tabular} & 75.21 & 75.61 & $75.41 \mathrm{~b}$ & 76.43 & 77.63 & 76.53 & 76.80 & 75.00 & \begin{tabular}{|l|}
75.37 \\
\end{tabular} & \begin{tabular}{|l|l|} 
& 75.99 \\
\end{tabular} & 76.60 & 76.29a & 76.42 & \begin{tabular}{|l|}
77.08 \\
\end{tabular} & 75.55 & 76.33 & 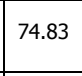 & 74.90 & 75.60 & 76.11 & $75.85 \mathrm{~b}$ \\
\hline \begin{tabular}{|l|} 
Beni- \\
Sweif 5 \\
\end{tabular} & $15 \frac{5}{3}$ & 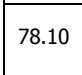 & \begin{tabular}{|l|}
77.43 \\
\end{tabular} & $\mid 76.40$ & 76.53 & 75.97 & \begin{tabular}{|l|l|} 
& 75.67 \\
\end{tabular} & 76.82 & 76.54 & 76.68a & 76.70 & 78.17 & 76.17 & \begin{tabular}{|l|l|} 
& 77.43 \\
\end{tabular} & 76.17 & 75.80 & \begin{tabular}{|l|}
76.34 \\
\end{tabular} & 77.13 & 76.74a & \begin{tabular}{|l|l|}
77.40 \\
\end{tabular} & $\mid 77.80$ & \begin{tabular}{|l|}
76.28 \\
\end{tabular} & 76.98 & \begin{tabular}{|l|l|} 
& 76.07 \\
\end{tabular} & 75.73 & 76.58 & 76.84 & 76.71a \\
\hline Mean & & 72.86 & \begin{tabular}{|l|l|} 
& 73.37 \\
\end{tabular} & \begin{tabular}{|l|l|}
71.47 \\
\end{tabular} & 72.51 & 70.98 & 71.66 & $71.77 \mathrm{~b}$ & $72.51 a$ & & 72.61 & \begin{tabular}{|l|l|}
73.97 \\
\end{tabular} & 72.09 & 72.69 & 71.05 & 71.37 & $71.92 \mathrm{~b}$ & $72.68 \mathrm{a}$ & & \begin{tabular}{|l|l|}
73.11 \\
\end{tabular} & 73.29 & 71.99 & 72.39 & 71.32 & 71.21 & $71.84 b$ & $72.59 \mathrm{a}$ & - \\
\hline General mea & & 73. & $11 a$ & 71. & $99 \mathrm{~b}$ & 71. & $32 \mathrm{~b}$ & & - & & 73. & $29 a$ & 72. & $39 b$ & 71. & $21 \mathrm{c}$ & & - & & 73.2 & & 72.1 & $19 b$ & 71. & $.27 \mathrm{c}$ & & - & \\
\hline Interaction & & & & & & N.S. & & & & & & & & & N.S. & & & & & & & & & N.S. & & & & \\
\hline
\end{tabular}

Means followed by the same letter (s) in each column within each treatment are not significant. 


\section{Effects on insect infestation}

\subsection{Cereal aphids}

The results illustrated in Table (6) showed percentages of four aphid species recorded on wheat field during 2009/10 and 2010/11 seasons. The bird cherry-oat aphid Rhopalosiphum padi was found with high density level (39.4\%) followed by greenbug Schizaphis garaminum (36.2\%). The least species were english grain aphid Sitobion avenae (16.1\%) and corn leaf aphid Rhopalosiphum maidis (8.3\%) .

Table 6. Average percentages of four aphid species recorded on wheat field during 2009/10 and 2010/11 seasons.

\begin{tabular}{|c|c|c|c|c|}
\hline \multirow{2}{*}{ Season } & \multicolumn{4}{|c|}{ Aphid species } \\
\cline { 2 - 5 } & $\boldsymbol{R}$. padi & S. graminum & S. avenae & R. maidis \\
\hline $\mathbf{2 0 0 9 / 1 0}$ & 34.0 & 37.7 & 18.2 & 10.1 \\
\hline $\mathbf{2 0 1 0 / 1 1}$ & 44.7 & 34.8 & 13.9 & 6.6 \\
\hline Mean & 39.4 & 36.2 & 16.1 & 8.3 \\
\hline
\end{tabular}

The results illustrated in Table (7) show the counts of cereal aphids recorded on the six wheat cultivars. These counts pointed out that, Beni-Sweif 5 was more favorable for the aphid infestation. It received higher counts (193.8 individuals/10 plants) followed by Sakha 94 and Gemmeiza 10 cultivars with an average of 138.6 and 127.9 individuals/10 plants, respectively. However, Beni-Sweif 4 and Sids 12 cultivars harbored lower counts (62.1 and 83.3 individuals/10 plants, respectively). The varietal effect as reported earlier by Abdel-Aziz et. al. (2002) Giza 163 and Sakha 69 were tolerant to all cereal aphids. El-Rawy et. al. (2007) revealed that, Gemmeiza 9 cultivar proved to be more tolerant against cereal aphids comparing to Gemmeiza 7cultivar. 
Table 7. Effect of seeding and nitrogen fertilizer rates on number of cereal aphids/10 plants of some wheat cultivars during the two successive seasons (2009/10 and 2010/11).

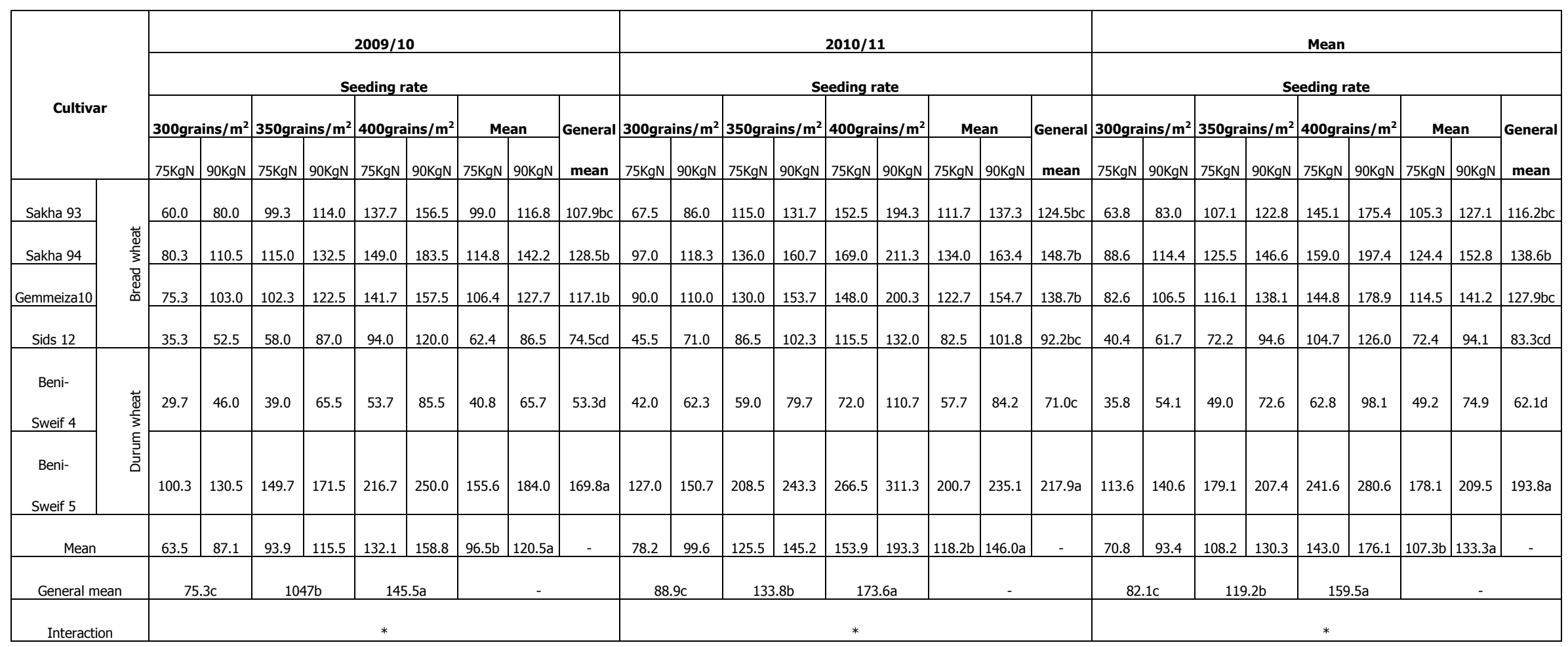

Means followed by the same letter (s) in each column within each treatment are not significant. 
The infestation of wheat plants by cereal aphids was significantly increased with the coincide increase of wheat seed rates. When the wheat crop was sown with a rate of $300 \mathrm{grains} / \mathrm{m}^{2}$, the mean number of aphids was 82.1 individuals/10 plants, while the mean number of aphids was reached to its maximum showing mean number of 159.5 individuals $/ 10$ plants when the seeding rate was increased to $400 \mathrm{grains} / \mathrm{m}^{2}$. These results are in agreement with those obtained by Ali and Ahmed (1996) and SIman (2002) who found that the population density of cereal aphids was increased with the increase of seed rate of wheat.

The highest rate of nitrogen $(90 \mathrm{Kg} / \mathrm{fed}$.) significantly increased the population of cereal aphids (133.3 individuals/10 plants) compared with $75 \mathrm{KgN} / \mathrm{fed}$ (107.3 individuals/10 plants). These results were confirmed with those obtained by Ali and Ahmed (1996), SIman (2002) and El-Rawy et. al. (2007).

\subsection{Leafminer}

As for Agromyza nigrella counts, the data found in Table (8) showed an opposite varietal trend against the infestation. Sakha 93 cultivar was more susceptible. It harbored 9.4 alive larvae/100 leaves which caused 12.8 mines followed by Gemmeiza 10 cultivar harbored 6.2 alive larvae/100 leaves and caused 8.5 mines. However, Beni-Sweif 4 and 5 durum cultivars harbored the least numbers of alive larvae/100 leaves (1.5 and 2.2) which caused 2.4 and 3.4 mines. The varietal effect is in harmony with El-Serwy (1996) who found that Giza 163 and Giza 165 were leas susceptible cultivars to leafminer. El-Rawy et. al. (2007) showed that Gemmeiza 7 cultivar was more susceptible to leafminer comparing with Gemmeiza 9.

The infestation of wheat plants by $A$. nigrella was significantly increased with the coincide increase of wheat seed rates. When wheat crop was sown with a rate of 300 grains $/ \mathrm{m}^{2}$, the mean number of alive larvae/100 leaves was 2.5 
Table 8. Effect of seeding and nitrogen fertilizer rates on number of $A$. nigrella mines and alive larvae/100 leaves of some wheat cultivars during the two successive seasons (2009/10 and 2010/11) .

\begin{tabular}{|c|c|c|c|c|c|c|c|c|c|c|c|c|c|c|c|c|c|c|c|c|c|c|c|c|c|c|c|c|}
\hline \multirow{5}{*}{ cultivar } & & \multicolumn{27}{|c|}{ No. of mines / 100 leaves } \\
\hline & & \multicolumn{9}{|c|}{$2009 / 10$} & \multicolumn{9}{|c|}{$2010 / 11$} & \multicolumn{9}{|c|}{ Mean } \\
\hline & & \multicolumn{9}{|c|}{ Seeding rate } & \multicolumn{9}{|c|}{ Seeding rate } & \multicolumn{9}{|c|}{ Seeding rate } \\
\hline & & \multicolumn{2}{|c|}{ 300grains $/ \mathrm{m}^{2}$} & \multicolumn{2}{|c|}{350 grains $/ \mathrm{m}^{2}$} & \multicolumn{2}{|c|}{ 400grains $/ \mathrm{m}^{2}$} & \multicolumn{2}{|c|}{ Mean } & \multirow{2}{*}{$\begin{array}{c}\text { General } \\
\text { mean }\end{array}$} & \multicolumn{2}{|c|}{300 grains $/ \mathrm{m}^{2}$} & \multicolumn{2}{|c|}{350 grains $/ \mathrm{m}^{2}$} & \multicolumn{2}{|c|}{ 400grains $/ \mathrm{m}^{2}$} & \multicolumn{2}{|c|}{ Mean } & \multirow{2}{*}{$\begin{array}{c}\text { General } \\
\text { mean }\end{array}$} & \multicolumn{2}{|c|}{300 grains $/ \mathrm{m}^{2}$} & \multicolumn{2}{|c|}{350 grains $/ \mathrm{m}^{2}$} & \multicolumn{2}{|c|}{ 400grains $/ \mathrm{m}^{2}$} & \multicolumn{2}{|c|}{ Mean } & \multirow{2}{*}{$\begin{array}{c}\text { General } \\
\text { mean }\end{array}$} \\
\hline & & \begin{tabular}{|l|}
$75 \mathrm{KgN}$ \\
\end{tabular} & $90 \mathrm{KgN}$ & $75 \mathrm{KgN}$ & $90 \mathrm{KgN}$ & $75 \mathrm{KgN}$ & $90 \mathrm{KgN}$ & $75 \mathrm{KgN}$ & $90 \mathrm{KgN}$ & & \begin{tabular}{|l|}
$75 \mathrm{KgN}$ \\
\end{tabular} & $90 \mathrm{KgN}$ & $75 \mathrm{KgN}$ & $90 \mathrm{KgN}$ & $75 \mathrm{KgN}$ & $90 \mathrm{KgN}$ & $75 \mathrm{KgN}$ & $90 \mathrm{KgN}$ & & \begin{tabular}{|l|}
$75 \mathrm{KgN}$ \\
\end{tabular} & $90 \mathrm{KgN}$ & $75 \mathrm{KgN}$ & $90 \mathrm{KgN}$ & $75 \mathrm{KgN}$ & $90 \mathrm{KgN}$ & $75 \mathrm{KgN}$ & $90 \mathrm{KgN}$ & \\
\hline Sakha 93 & & 8.0 & 12.0 & 14.0 & 19.0 & 18.0 & 23.0 & 13.3 & 18.0 & $15.7 \mathrm{a}$ & 4.3 & 5.5 & 7.7 & 13.5 & 10.7 & 17.2 & 7.6 & 12.1 & $9.9 \mathrm{a}$ & 6.1 & 8.7 & 10.8 & 16.2 & 14.3 & 20.1 & 10.4 & 15.1 & $12.8 \mathrm{a}$ \\
\hline Sakha 94 & 贾 㦴 & 3.0 & 5.7 & 5.5 & 8.0 & 7.3 & 11.3 & 5.3 & 8.3 & $6.8 \mathrm{c}$ & 2.7 & 3.3 & 4.3 & 7.0 & 5.5 & 10.3 & 4.2 & 6.9 & $5.6 \mathrm{c}$ & 2.8 & 4.5 & 4.9 & 7.5 & 6.4 & 10.8 & 4.7 & 7.6 & $6.2 \mathrm{c}$ \\
\hline Gemmeiza10 & 商 & 4.0 & 7.0 & 8.5 & 10.7 & 11.5 & 14.0 & 8.0 & 10.6 & $9.3 \mathrm{~b}$ & 3.3 & 4.5 & 5.3 & 9.0 & 9.0 & 14.7 & 5.9 & 9.4 & $7.7 \mathrm{~b}$ & 3.6 & 5.7 & 6.9 & 9.8 & 10.2 & 14.3 & 6.9 & 10.0 & $8.5 \mathrm{~b}$ \\
\hline Sids 12 & & 2.5 & 5.1 & 5.0 & 7.3 & 7.0 & 10.7 & 4.8 & 7.7 & $6.3 \mathrm{c}$ & 2.0 & 3.0 & 3.3 & 5.7 & 5.7 & 9.5 & 3.7 & 6.1 & $4.9 c$ & 2.2 & 4.0 & 4.1 & 6.5 & 6.3 & 10.1 & 4.2 & 6.9 & $5.6 c$ \\
\hline $\begin{array}{l}\text { Beni- } \\
\text { Sweif } 4\end{array}$ & छ मू & 0.5 & 2.0 & 2.0 & 4.7 & 3.0 & 6.0 & 1.8 & 4.2 & $3.0 \mathrm{~d}$ & 0.3 & 1.5 & 1.3 & 2.7 & 2.0 & 3.7 & 1.2 & 2.6 & $1.9 \mathrm{~d}$ & 0.4 & 1.7 & 1.6 & 3.7 & 2.5 & 4.8 & 1.5 & 3.4 & $2.4 \mathrm{~d}$ \\
\hline $\begin{array}{l}\text { Beni- } \\
\text { Sweif } 5\end{array}$ & 咅 & 1.3 & 2.5 & 3.0 & 5.5 & 5.0 & 7.5 & 3.1 & 5.2 & $4.2 d$ & 1.0 & 2.3 & 2.0 & 3.5 & 3.0 & 4.3 & 2.0 & 3.4 & $2.7 d$ & 1.1 & 2.4 & 2.5 & 4.5 & 4.0 & 5.9 & 2.5 & 4.3 & $3.4 \mathrm{~d}$ \\
\hline Mean & & 3.2 & 5.7 & 6.3 & 9.2 & 8.6 & \begin{tabular}{|l|}
12.1 \\
\end{tabular} & $6.0 \mathrm{~b}$ & $9.0 \mathrm{a}$ & - & 2.3 & 3.4 & 4.0 & 6.9 & 6.0 & 10.0 & $4.1 \mathrm{~b}$ & $6.8 \mathrm{a}$ & - & 2.7 & 4.6 & 5.1 & 8.0 & 7.3 & 11.0 & $5.0 \mathrm{~b}$ & $7.9 \mathrm{a}$ & - \\
\hline General mear & & 4.5 & & 7. & $8 \mathrm{~b}$ & 10. & $4 a$ & & - & & 2.9 & & 5. & & 8. & $0 \mathrm{a}$ & & - & & 3.7 & & 6.6 & $6 \mathrm{~b}$ & 9.2 & $2 a$ & & - & \\
\hline Interaction & & & & & & $*$ & & & & & & & & & $*$ & & & & & & & & & $*$ & & & & \\
\hline & & & & & & & & & & & & No. of & alive la & rvae / 1 & 100 leav & & & & & & & & & & & & & \\
\hline Sakha 93 & & 5.5 & 9.0 & 10.3 & 14.5 & 14.3 & 19.0 & 10.0 & 14.2 & $12.1 \mathrm{a}$ & 2.7 & 4.0 & 5.3 & 8.6 & 7.7 & 12.5 & 5.2 & 8.4 & $6.8 \mathrm{a}$ & 4.1 & 6.5 & 7.8 & 11.5 & 11.0 & 15.7 & 7.6 & 11.3 & $9.4 a$ \\
\hline Sakha 94 & 召 芴 & 2.0 & 3.7 & 4.0 & 6.0 & 4.5 & 9.5 & 3.5 & 6.4 & $5.0 \mathrm{c}$ & 1.7 & 2.5 & 3.3 & 4.7 & 4.7 & 6.5 & 3.2 & 4.6 & $3.9 \mathrm{c}$ & 1.8 & 3.1 & 3.6 & 5.3 & 4.6 & 8.0 & 3.3 & 5.5 & $4.4 \mathrm{c}$ \\
\hline Gemmeiza10 & 部 & 2.7 & 5.0 & 6.5 & 8.0 & 8.0 & 11.7 & 5.7 & 8.2 & $7.0 \mathrm{~b}$ & 2.3 & 3.7 & 4.0 & 6.3 & 6.3 & 10.0 & 4.2 & 6.7 & $5.5 \mathrm{~b}$ & 2.5 & 4.3 & 5.2 & 7.1 & 7.1 & 10.8 & 4.9 & 7.4 & $6.2 \mathrm{~b}$ \\
\hline Sids 12 & & 1.3 & 3.3 & 3.0 & 5.0 & 5.0 & 8.3 & 3.1 & 5.5 & $4.3 c$ & 1.3 & 2.0 & 2.3 & 3.5 & 3.7 & 5.5 & 2.4 & 3.7 & $3.1 \mathrm{c}$ & 1.3 & 2.6 & 2.6 & 4.2 & 4.3 & 6.9 & 2.7 & 4.6 & $3.7 \mathrm{c}$ \\
\hline $\begin{array}{l}\text { Beni- } \\
\text { Sweif } 4\end{array}$ & 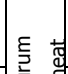 & 0.3 & 0.8 & 1.5 & 2.7 & 1.5 & 3.7 & 1.1 & 2.4 & $1.8 \mathrm{~d}$ & 0.0 & 0.7 & 0.7 & 2.0 & 1.3 & 2.3 & 0.7 & 1.7 & $1.2 \mathrm{~d}$ & 0.2 & 0.7 & 1.1 & 2.3 & 1.4 & 3.0 & 0.9 & 2.0 & $1.5 \mathrm{~d}$ \\
\hline $\begin{array}{l}\text { Beni- } \\
\text { Sweif } 5\end{array}$ & 咅 & 0.7 & 1.5 & 2.3 & 3.5 & 3.0 & 5.3 & 2.0 & 3.4 & $2.7 \mathrm{~d}$ & 0.7 & 1.3 & 1.3 & 2.7 & 2.0 & 3.0 & 1.3 & 2.3 & $1.8 \mathrm{~d}$ & 0.7 & 1.4 & 1.8 & 3.1 & 2.5 & 4.1 & 1.6 & 2.8 & $2.2 \mathrm{~d}$ \\
\hline Mean & & 2.1 & 3.9 & 4.6 & 6.6 & 6.1 & 9.6 & $4.2 \mathrm{~b}$ & $6.7 a$ & - & 1.5 & 2.4 & 2.8 & 4.6 & 4.3 & 6.6 & $2.8 \mathrm{~b}$ & $4.6 \mathrm{a}$ & - & 1.8 & 3.1 & 3.7 & 5.6 & 5.2 & 8.1 & $3.5 \mathrm{~b}$ & $5.6 \mathrm{a}$ & - \\
\hline General mear & & 3.0 & $\underline{O c}$ & 5. & $6 \mathrm{~b}$ & 7. & $9 a$ & & - & & 2.0 & & 3. & & 5. & $5 \mathrm{a}$ & & - & & 2.5 & $5 c$ & 4.6 & $6 \mathrm{~b}$ & 6.7 & $7 \mathrm{a}$ & & - & \\
\hline Interaction & & & & & & $*$ & & & & & & & & & $*$ & & & & & & & & & $*$ & & & & \\
\hline
\end{tabular}

Means followed by the same letter (s) in each column within each treatment are not significant. 
which caused 3.7 mines, while the mean number of alive larvae/100 leaves was 6.7 which caused 9.2 mines when increasing the seed rate to $400 \mathrm{grains} / \mathrm{m}^{2}$. These results are in agreement with those obtained by SIman et. al.. (2002).

The mean numbers of mines and alive larvae were significantly lower on plants receiving $75 \mathrm{KgN} /$ fed than those receiving $90 \mathrm{KgN} /$ fed showing values of 5.0 mines and 3.5 larvae/100 leaves and 7.9 mines and 5.6 larvae/100 leaves, respectively. These results confirmed by those obtained by Slman et. al. (2002) and El-Rawy et. al. (2007).

Data illustrated in Tables (1 to 5 ) pointed out that, the interaction between the tested cultivars, seeding rates and $\mathrm{N}$ rates did not show any significant effect on all studied wheat traits. These results are in agreement with those obtained by ElGharieb et. al. (1998), El-Karamity (1998), Munir et. al. (2001) and El-Rawy et. al. (2007). On the contrary, the interaction revealed significant effects on the aphid and leafminer infestation as shown in Tables (7and 8).

From these results, it could be concluded that sowing Sids 12 cultivar or BeniSweif 4 cultivar under seeding rate of 300 grains $/ \mathrm{m}^{2}$ and applying $90 \mathrm{KgN} /$ fed gave the highest grain yield/fed and least infestation by cereal aphids and leafminer, in both beard wheat commercial cultivars as well as durum wheat commercial cultivars. 


\section{REFERENCES}

1. Abdel-Aziz, M.A., A.A. Abdel-Alim, N.A. Abdel-Aziz and G. A. Morsi 2002. Susceptibility of different wheat varieties to infestation to cereal aphids with reference to safe control approach. $2^{\text {nd }}$ Inter. Conf., PI. Port. Res. Inst., Cairo, Egypt, 21-24 Dec. 2:685-690.

2. Abdel-Nour, N.A.R. and H.S.A. Fateh 2011. Influence of sowing date and nitrogen fertilization on yield and its components in some bread wheat genotypes. Egypt. J. Agric. Res. 89 (4): 1413-1433.

3. Ali, A.G. and A.A.S. Ahmed 1996. Effect of plant density and nitrogen fertilization on the infestation of wheat plants with cereal aphid. Assiut J. Agric. Sci. 27(2): 119-124.

4. A.O.A.C. 2000. Official Methods of Analysis of the Association of Official Analytical Chemists, 17th Ed, Published by A.O.A.C. Arlingto, Virgina, USA.

5. El-Ghareib, E.A., A.M.Azab, M.M. El-Monoufi and G.A. El-Shaarawy 1998. Effect of nitrogen fertilizer and seeding rate on yield and yield components of some new wheat cultivars. Al-Azhar J. Agric. Res. 27: 31-46.

6. El-Karamity, A.E. 1998. Response of some wheat cultivars to seeding and $\mathrm{N}$ fertilization rates. Mansoura J. Agric. Sci. 23(2): 643-655.

7. El-Naggar, G.R. 2003. Yield and quality of some spring wheat genotypes subjected to different nitrogen fertilizer rates. Assiut J. Agric. Sci. 34 (2): 43-63.

8. El-Rawy, A.M., M.A. Khaled and S.M. Osman 2007. Impact of NPK fertilizers, wheat varieties and their interactions on the infestation with cereal aphids and leafminer, yield and its components. J.Agric. Sci. Mansoura Univ. 32 (4): 31133122.

9. El-Serwy, S.A. 1996. Evaluation of certain wheat varieties for susceptibility to infestation with the leafminer Agromyza nigripes (Meigen) (Diptera : Agromyzidae) and grain yield at El-Gemmeiza and Sids regions. Bull. Ent. Soc. Egypt 74: 139148. 
10. Hefni, E.S.H.M., A.A. El-Kholany and N.A. Abdel-Hamid 1979. Effect of seed rates and nitrogen fertilizer levels on the yield, yield components, chemical content and technological properties of Egyptian wheat. II: Chemical content and technological properties. First Conf. Agric. Res. Center, 22-29 May vol. I: 54-60.

11. Mousa, A. 2006. Effect of different seeding rate on grain yield of two released cultivars. Annual Workshop for Winter Field Crops, 8-9 Nov. : 9.

12. Munir, A.A.E., I.E.M.A. El-Beially, H.Y.M. Yousif and M.S. El-Karmany 2001. Effect of irrigation intervals and seeding and nitrogen fertilizer rates on yield and yield components of wheat. Al-Azhar J. Agric. Res. 34 (Dec.): 69-83.

13. Sharaan, A.N. and F.S. Abdel-Samie 1999. Effect of seeding rates and N fertilization levels on growth and yield of two wheat varieties (Triticum aestivum L.). Annals Agric. Sci. Ain Shams Univ., Cairo 44 (2): 589-601.

14. SIman, F.A.A. 2002. Influence of some agricultural practices on the infestation of wheat crop by cereal aphids in Upper Egypt. Assiut J. Agric. Sci. 33 (3): 1-12.

15. Slman, F.A.A., H.A. Mohamed, H.E.M. Salem and K.H. El-Laithy 2002. Effect of some agricultural practices on wheat infestation by leafminer Agromyza nigripes (Meigen) (Diptera: Agromyzidae) in Sohag Governorate Upper Egypt. Egypt. J. Appl. Sci. 17 (10): 428-439.

16. Steel, R.G., J.H. Torrie and D. A.Dickey 1997. Principles and Procedures of Statistics : A Biometrical Approach. 3rd ed. McGraw-Hill Book Co., New York, USA, 666pp.

17. Swelam, A.A. 2008. Mean performance, correlation and path coefficient analysis for grain yield and its components of four bread wheat cultivars grown under four nitrogen levels. Egypt. J. Agric. Res. 86 (2): 561-573. 
تأثير بعض معدلات التقاوى والتسميد النيتروجينى على محصول الحبوب والإصابة الطبيعية بالحشرات لبعض أصناف القمح عئم

$$
\begin{aligned}
& \text { محمد عبد الكريم خالد } 1 \text { عادل محمد الراوي2 } \\
& \text { 1- قسم بحوث القهح - دعهز بحوث المحاصيل الحقلية - مركز البحوث الزراعية - جيزة - مصر. } \\
& \text { 2- معرة بحوث وقاية النباتات - مركز البحوث الزراعية - الدقي - جيزة - مصر. }
\end{aligned}
$$

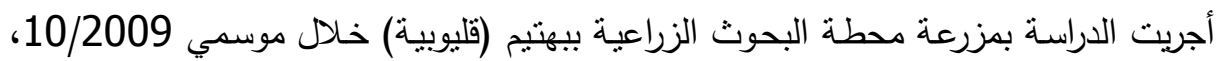

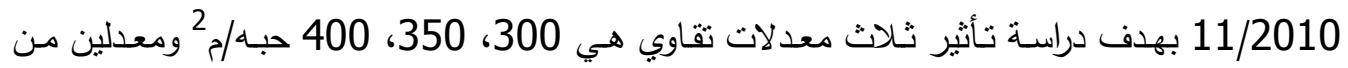

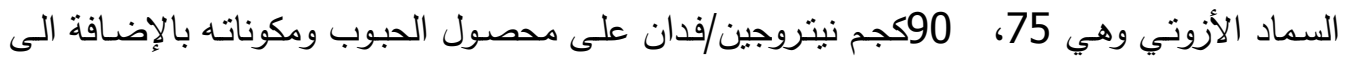

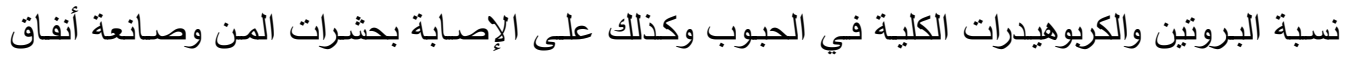

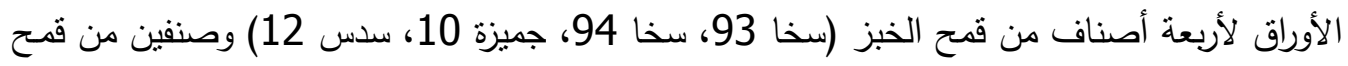
المكرونة (بني سويف 4، بني سويف 5).

أظهرت النتائج أن الصنف بني سويف 4 إحتاج لفترات أطول نسبياً سواء لبلوغ طور طرد السنابل (89.5 يوم) أو طور النضج (140.5 يوم). وأن نباتات الصنف بني سويف 5 كانت الأطول

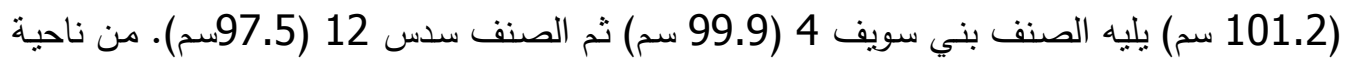

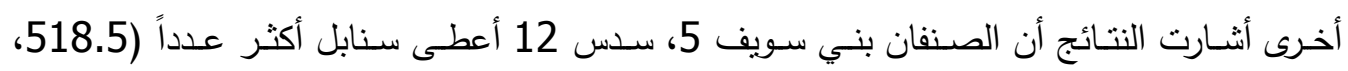

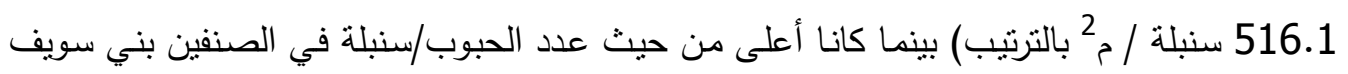

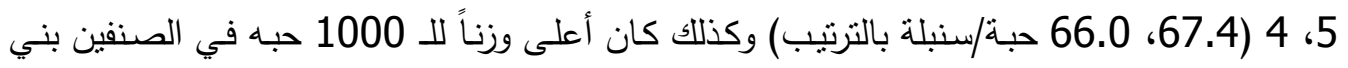

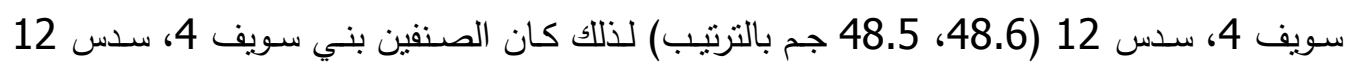

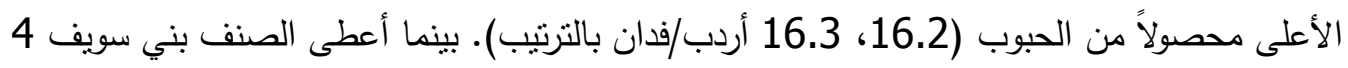

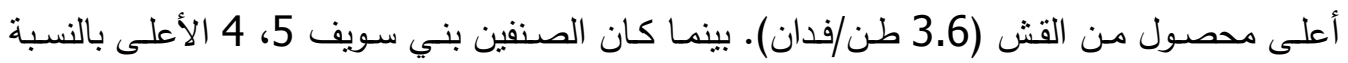

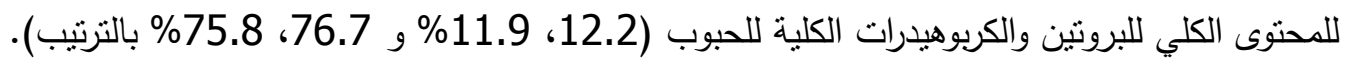

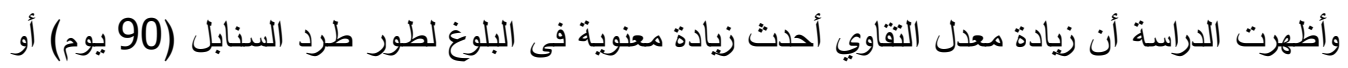

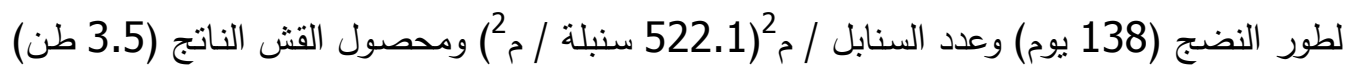

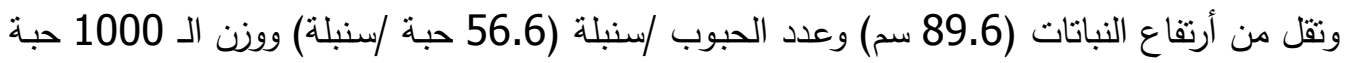
(42.9 جم) ومحصول الحبوب الناتج (15.5 أردب/فدان) وكذلك تقل من نسبة البروتين والكربوهيدرات

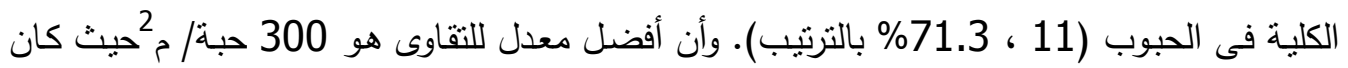




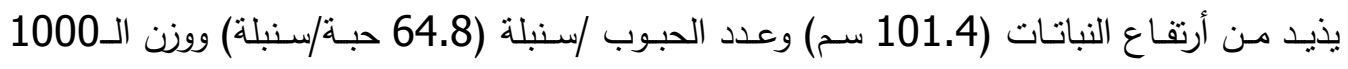

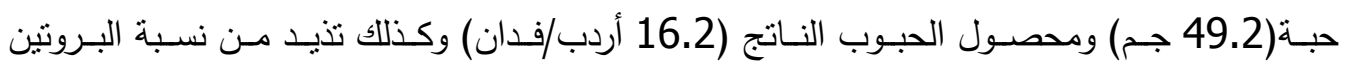

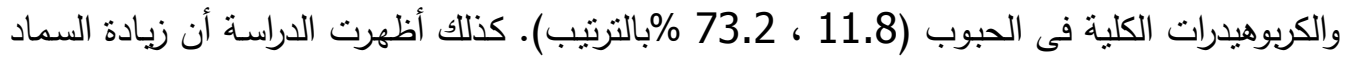

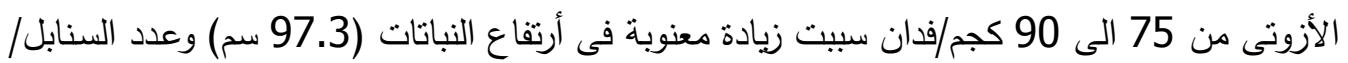

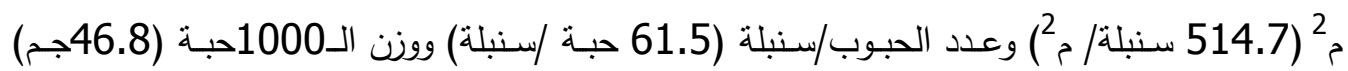
ومحصول الحبوب (16.2 أردب/فدان) ومحصول القش(3.4 طن/فدان) وكذلك على زيادة فى نسبة

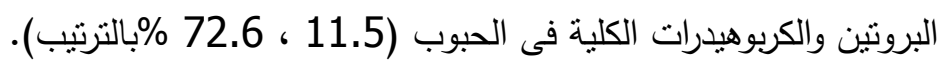

أما بالنسبة للإصابة بالحشرات فقد أظهرت النتائج أن صنفى سويف 4 وسدس 12 كانا أقل

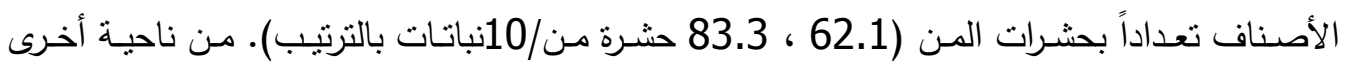

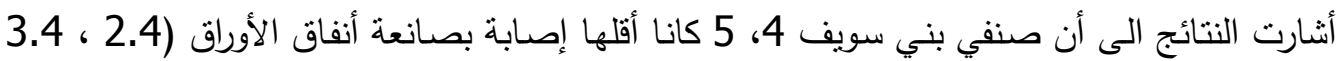

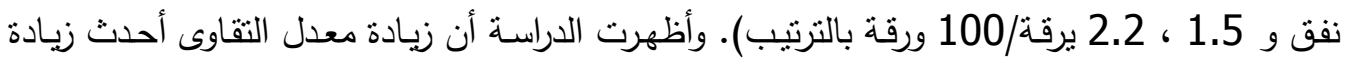

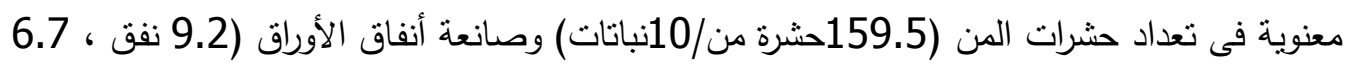

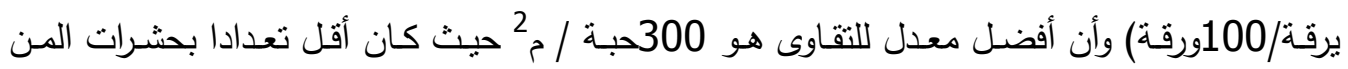

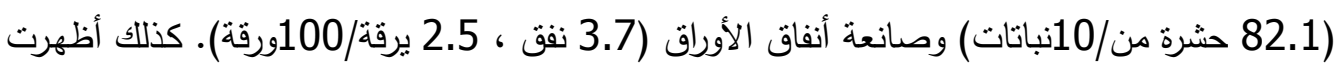

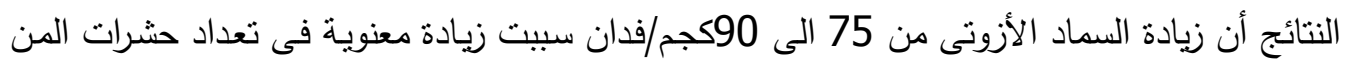

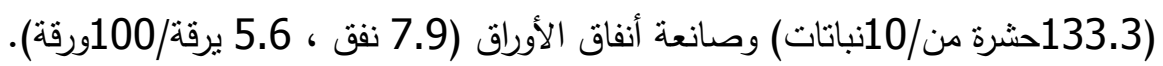
وعموماً نتير النتائج إلى أن زراعة الصنف سدس 12 (خبز) أو بنى سويف 4 (مكرونة)

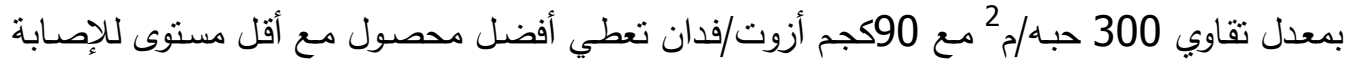
سواء بالمن أو بصانعة أنفاق الأوراق. 